\title{
Geomagnetic response to solar and interplanetary disturbances
}

\author{
Elena Saiz ${ }^{1, *}$, Yolanda Cerrato ${ }^{1}$, Consuelo $\mathrm{Cid}^{1}$, Venera Dobrica ${ }^{2}$, Pavel Hejda ${ }^{3}$, Petko Nenovski ${ }^{4}$, Peter Stauning ${ }^{5}$, \\ Josef Bochnicek ${ }^{3}$, Dimitar Danov ${ }^{6}$, Crisan Demetrescu ${ }^{2}$, Walter D. Gonzalez ${ }^{7}$, Georgeta Maris ${ }^{2}$, Dimitar Teodosiev ${ }^{6}$, \\ and Fridich Valach ${ }^{8}$ \\ 1 Space Research Group-Space Weather, Departamento de Física, Universidad de Alcalá, Madrid, Spain \\ *Corresponding author: e-mail: elena.saiz@uah.es \\ 2 Institute of Geodynamics, Romanian Academy, Bucharest, Romania \\ 3 Institute of Geophysics of the ASCR, Prague, Czech Republic \\ 4 National Institute for Geophysics, Geodesy and Geography, Bulgarian Academy of Sciences, 1113 Sofia, Bulgaria \\ 5 Danish Meteorological Institute, Copenhagen, Denmark \\ 6 Institute for Space Research and Technologies, Bulgarian Academy of Sciences, 1113 Sofia, Bulgaria \\ 7 Instituto Nacional de Pesquisas Espaciais (INPE), 12245-970 Sao Jose dos Campos, Sao Paulo, Brazil \\ 8 Geomagnetic Observatory, Geophysical Institute, Slovak Academy of Sciences, Hurbanovo, Slovakia
}

Received 1 June 2012 / Accepted 23 June 2013

\begin{abstract}
The space weather discipline involves different physical scenarios, which are characterised by very different physical conditions, ranging from the Sun to the terrestrial magnetosphere and ionosphere. Thanks to the great modelling effort made during the last years, a few Sun-to-ionosphere/thermosphere physics-based numerical codes have been developed. However, the success of the prediction is still far from achieving the desirable results and much more progress is needed. Some aspects involved in this progress concern both the technical progress (developing and validating tools to forecast, selecting the optimal parameters as inputs for the tools, improving accuracy in prediction with short lead time, etc.) and the scientific development, i.e., deeper understanding of the energy transfer process from the solar wind to the coupled magnetosphere-ionosphere-thermosphere system. The purpose of this paper is to collect the most relevant results related to these topics obtained during the COST Action ES0803. In an end-to-end forecasting scheme that uses an artificial neural network, we show that the forecasting results improve when gathering certain parameters, such as X-ray solar flares, Type II and/or Type IV radio emission and solar energetic particles enhancements as inputs for the algorithm. Regarding the solar wind-magnetosphere-ionosphere interaction topic, the geomagnetic responses at high and low latitudes are considered separately. At low latitudes, we present new insights into temporal evolution of the ring current, as seen by Burton's equation, in both main and recovery phases of the storm. At high latitudes, the PCC index appears as an achievement in modelling the coupling between the upper atmosphere and the solar wind, with a great potential for forecasting purposes. We also address the important role of small-scale field-aligned currents in Joule heating of the ionosphere even under non-disturbed conditions. Our scientific results in the framework of the COST Action ES0803 cover the topics from the short-term solar-activity evolution, i.e., space weather, to the long-term evolution of relevant solar/heliospheric/magnetospheric parameters, i.e., space climate. On the timescales of the Hale and Gleissberg cycles (22- and 88-year cycle respectively) we can highlight that the trend of solar, heliospheric and geomagnetic parameters shows the solar origin of the widely discussed increase in geomagnetic activity in the last century.
\end{abstract}

Key words. solar activity - interplanetary medium - indices - ionosphere (general) - ring current

\section{Introduction}

Today, ground-based and space-borne solar observations reveal that a geomagnetic storm can be regarded as an event in which disturbances are triggered by solar eruptions. These features, that have their origin in the magnetic activity of the Sun, propagate through interplanetary space and interact with the terrestrial magnetosphere subsequently affecting the near-Earth space environment and the upper atmosphere.

These disturbances have the potential to influence the performance and reliability on many space- and ground-based technological systems at risk, causing temporary disruption of the system or even damage in the case of severe disturbances. Although the technology was affected only by the most severe ones in the past, nowadays our society demands more sophisticated technology, therefore may become more vulnerable even at less severe disturbances. In this way, the space weather discipline was born in the late 1900s to forecast the adverse con- ditions at terrestrial environment due to solar disturbances. The task of forecasting space weather deals with four major scientific disciplines: solar physics, interplanetary physics, magnetospheric physics and ionospheric physics, which need to be synthesised and integrated. Given the interdisciplinary nature of the issue, initiatives such as the Actions supported by the European Cooperation in Science and Technology (COST) provide the best framework for this task.

In fact, the COST Action ES0803, "Developing Space Weather Products and Services in Europe" (http://www. costes0803.noa.gr/), was born to form an interdisciplinary network between European scientists dealing with different issues of Geospace, as well as warning system developers and operators. Many activities have been carried out by the participants of this COST Action that should be shared with the space weather community. In particular, this paper presents a summary of results pertaining to the interaction of the solar wind with the magnetosphere and the energy transfer inside the 
magnetosphere-ionosphere system. The scope of the paper is wide since it deals with solar physics, interplanetary physics, magnetospheric physics and aeronomy. The aim of this paper is to review those advances made in the framework of the COST Action ES0803 on geomagnetic response to solar and interplanetary disturbances topic that could be useful for the space weather community.

The COST Action ES0803 is not a unique case of interdisciplinary space weather networks. Beyond Europe, other approaches can be also found, mainly in the modelling discipline, which try to overcome the forecasting scenario joining different approaches for every stage between the Sun and the ionosphere/thermosphere. A good example of this approach is the CISM (Center for Integrated Space Weather Modeling) project (Hughes \& Hudson 2004), which entails interactively coupling together, like links in a chain, separate physics-based numerical codes in an end-to-end (Sun-to-ionosphere/thermosphere) numerical code to predict space weather. A similar approach is the one of the Space Weather Modeling Framework (SWMF), which provides a high-performance flexible framework for physics-based space weather simulations, and various space physics applications as well (Tóth et al. 2005). We should also mention, as a different style, the Community Coordinated Modeling Center (http://ccmc.gsfc.nasa.gov/index.php), which is a multi-agency partnership to enable, support and perform the research and development for autonomous space weather models. A review of the existing networks is beyond the scope of this paper, but the above examples illustrate the collaborative scenario in the space weather community. However, these models and the links between them need to be validated by external parties to be reliable and improved on the base of the results obtained to achieve a successful real-time model for the prediction of space weather disturbances, in particular for the most severe ones.

Besides the results of an external validation process of the existing models, model developers are aware of some gaps in their own models, and several open questions in different fields of the science concerning the Sun-Earth connection are recognised by the scientific community. To step forward on these issues, more collaborative studies need to be undertaken.

This paper summarises the results obtained by some studies performed during COST Action ES0803, which focus on the response of the terrestrial environment to solar and interplanetary disturbances. In the next section we give an overview of the long-term evolution of the reconstructed solar outputs and the geomagnetic response based on several indices as indicators of different magnetospheric currents. Section 3 is devoted to forecast geomagnetic disturbances, as seen by the $K p$ index, from solar observations by means of artificial neural networks, in particular the geoeffectiveness of coronal mass ejection (CME) events using X-ray solar flares as proxies for CME launches. In Section 4, some advances in the solar wind-magnetospheric response coupling at both high and low latitudes are highlighted, which will be useful for space weather forecasting purposes. Finally, Section 5 completes the paper.

\section{Long-term evolution of the geomagnetic response}

Complex interaction of the solar outputs - electromagnetic radiation, solar wind, interplanetary magnetic field (IMF) - with the near-Earth space environment modifies the electric currents of the environment, producing geomagnetic field variations which can be detected from the magnetosphere down to the ground. While the electromagnetic solar radiation originates the charged particles of the ionosphere (contributing to the Sq current system responsible for the regular diurnal magnetic field variations), the particle and magnetic field outputs of the Sun interact with the magnetosphere, producing current systems that are sources of the irregular variations called the disturbance magnetic field that characterises the so-called geomagnetic activity (e.g., Rangarajan 1989; Campbell 2003). The geomagnetic activity is characterised through geomagnetic indices, designed as proxies for various current systems in the magnetosphere and ionosphere, such as Dst for geomagnetic storm-time activity, $A E$ for the auroral electrojet, $P C$ for the polar cap currents, $a a, A p, I D V$ for geomagnetic activity at mid-latitudes, etc. They are elaborated by combining data of the geomagnetic field measured by a station network spread around the world and in recent years they are important parameters in space weather analysis; the indices are usually used to detect, describe and quantify space weather events, especially for times prior to space era when in situ monitoring of space weather was missing. The coarse time resolution of some "traditional" indices, like $K p, a a$, of $3 \mathrm{~h}$, makes them poor tools for assessing many impacts of space weather. However, other indices have 15-minute values $(P C)$ and even 1-minute values (the new 1-minute $D s t$ ). A detailed description of the numerous geomagnetic indices is out of the scope of this paper, but can be found, for instance, in Menvielle (2011).

On the other hand, solar activity is also represented by several solar indices. Two classical indices are related to the electromagnetic output of the Sun: the $10.7 \mathrm{~cm}$ solar radio flux (F10.7), whose record extends back to 1947, and the sunspot number $(R)$, the longest series of solar observation and most commonly used solar proxy.

The study of geomagnetic activity through indices has long contributed to progress in solar-terrestrial science because long geomagnetic time series recorded at the terrestrial surface have provided means to characterise the Sun-Earth interaction at times prior to the space era.

Studies of the long-term evolution of solar activity are reviewed by Kuklin (1976). A 22-year variation referred to as the "Hale-cycle-related" or "solar-magnetic-cycle-related" variation (MC) seems to be linked to the magnetic field of the Sun and its changing polarity. On the other hand, the Gleissberg cycle (GC; also referred to as the "secular" or "80-90-year" cycle) manifests itself as a modulation of the amplitude and frequency of the 11-year solar cycle. It is empirically defined but its physical meaning is not yet clear. There is a long list of studies on long-term evolution of the geomagnetic activity and its relationship with the solar variability, such as those published by Feynman \& Crooker (1978), Svalgaard (1978), Cliver et al. (1996), Andreasen (1997), Cliver et al. (1998), Stamper et al. (1999), Lockwood et al. (1999), Richardson et al. (2002), Mursula et al. (2001), Svalgaard et al. (2003), Echer et al. (2004), Svalgaard et al. (2004), Mursula et al. (2004), Le Mouël et al. (2005), Clilverd et al. (2005), Svalgaard \& Cliver (2005), Svalgaard \& Cliver (2007), Rouillard et al. (2007), Lockwood et al. (2009), Feynman \& Ruzmaikin (2011), Du (2011), Richardson \& Cane (2012a, 2012b).

The information that can be derived from the long-term characteristics is highly important in terms of space climate (e.g., Eddy 1977). For example, Lockwood et al. (1999) based on a study by Stamper et al. (1999) on the connection between the geomagnetic activity and solar causes for the solar cycles 20-22 (1964-1996) - analysed the solar causes of the 
long-term increase in geomagnetic activity observed in the $a a$ index since 1900, concluding that the increased geomagnetic activity was caused by a rise in the interplanetary magnetic field and they inferred an increase of about $100 \%$ of the solar open flux. However, the choice between various coupling functions describing the energy transfer between the solar wind and magnetosphere remains a difficult matter (Finch \& Lockwood 2007) and much effort is needed.

Within the frame of the COST Action, two papers have been published on the long-term behaviour of the solar/heliosphere/magnetosphere environment: Demetrescu \& Dobrica (2008) and Demetrescu et al. (2010). Annual means of measured and reconstructed solar, heliospheric and magnetospheric parameters were used to characterise the space climate through the long-term evolution of the relevant parameters, and to infer solar-activity signatures on the timescales of the Hale and Gleissberg cycles in the three environments.

Figure 1 shows the time series of the available instrumental annual means of the parameters used to characterise heliosphere-magnetosphere environment. The ordinate scale of each plot was chosen to yield comparable amplitudes to facilitate a first, visual comparison among the parameters. Vertical dashed lines mark epochs of solar minimum. From top to bottom, a first plot group includes measurements obtained by instruments on board spacecraft located at $1 \mathrm{AU}$, that is, the interplanetary magnetic field $(B)$, the solar wind velocity $(V)$, the proton density $(N)$ and the total solar irradiance (TSI); a second plot group concerns several geomagnetic indices related to various magnetospheric currents, namely $a a$, Inter-Diurnal Variability (IDV), Geomagnetic Auroral Electrojet $(A E)$, Polar Cap $(P C)$ and Disturbance Storm Time (Dst) indices; and a third plot group encompasses the solar activity measured from the terrestrial surface, as expressed by the sunspot number $(R)$ and the Galactic cosmic-ray flux $(C R)$, expressed by data from the Climax neutron monitor.

Available open solar flux $(F s)$, modulation strength $(\Phi)$, cosmic-ray flux $(C R)$, total solar irradiance $(T S I)$ data reconstructed back to 1700 , solar wind parameters (velocity and density) and the magnitude of the interplanetary magnetic field at 1 $\mathrm{AU}$, reconstructed back to 1870 , as well as the time series of the geomagnetic activity indices $a a$ and $I D V$, going back to 1870 , have also been considered. The sources of data are given in Table 1, along with the time range of each data set.

All parameters studied show solar-cycle variations, with peculiarities depending on the particular parameter considered (peak $B$ after the solar maximum and Gnevyshev gap, high $V$ in the descending phase of a cycle, the double peak of $a a$ in a cycle, the general anti-correlation of $V / N, a a / D s t, C R / R)$. Simple filtering procedures (successive 11-, 22- and 88-year running averages and differences between them) were employed to detect Hale and Gleissberg signals in the 11-year smoothed time series. Scaling by the standard deviation from the average value for the common interval covered by the data shows that the long-discussed variation in the 20th century (a pronounced increase since $\sim 1900$, followed by a depression in the 1960 s and an increase peaking at 1987 ) seen in the 11-year averages of the analysed parameters (Fig. 2, top panel) is a result of the superposition in data of solar-activity signatures at Hale and Gleissberg cycles timescales (Fig. 2, middle and bottom panels, respectively). This can be seen at first sight in case of the magnetic cycle, that presents maxima and minima coincident in time with the 11-year ones, and only to 1950 in case of the $\mathrm{GC}$, because of lost information at the ends of the time series

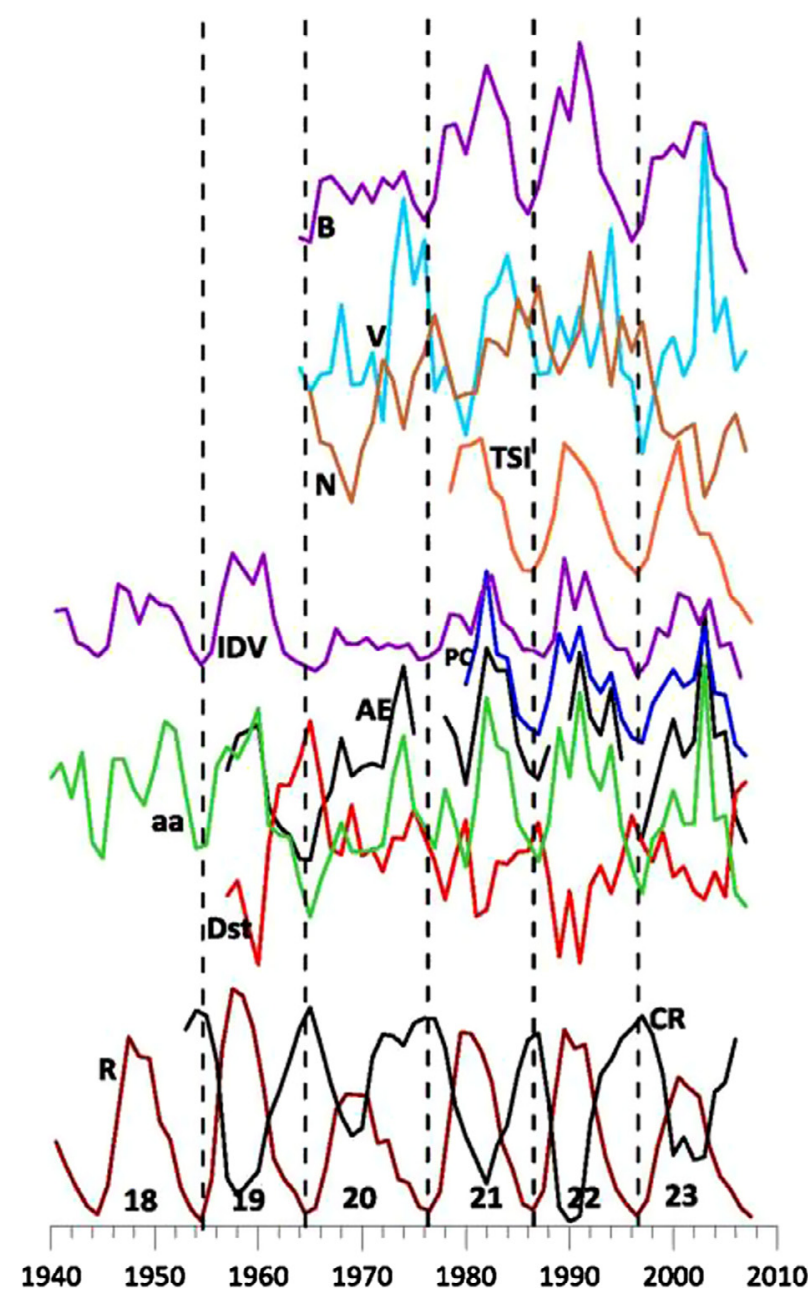

Fig. 1. Time series of annual means of instrumental data: solar outputs at $1 \mathrm{AU}$, including $B(\mathrm{nT}), V\left(\mathrm{~km} \mathrm{~s}^{-1}\right), N\left(\mathrm{~cm}^{-3}\right)$ and $T S I$ $\left(\mathrm{W} \mathrm{m}^{-2}\right.$ ), geomagnetic indices, i.e., $a a(\mathrm{nT}), I D V(\mathrm{nT}), D s t(\mathrm{nT}), A E$ (nT) and $P C$ and cosmic-ray flux, i.e., $C R$, based on measurements from the Climax neutron monitor $\left(10^{5}\right.$ counts $\left.\mathrm{h}^{-1}\right)$, compared with the sunspot number time series $(R)$. The ordinate axes (not shown) are arbitrarily set to yield comparable amplitudes for all parameters. Vertical dashed lines mark solar minima. Solar cycles are numbered. (From Demetrescu et al. 2010).

due to the 88-year averaging. At each of the two timescales the signals are quite similar for all parameters studied, pointing to a common pacing source: the solar dynamo. Indeed, the variability of magnetosphere and ionosphere, as shown by the geomagnetic indices, is a result of the variability of the solar wind and IMF interacting with the magnetosphere and ionosphere, as shown at $1 \mathrm{AU}$ by the speed and particle density and by the strength of IMF. In turn, solar wind and IMF variability is a result of the variability of processes in the Sun and its corona, having as ultimate source the convection zone with its dynamo. Of course, this causal chain implies certain delays between its elements, delays that are less and less significant as the timescale increases, making the long-term behaviour similar for all studied parameters. Details regarding small phase differences and small amplitude wiggles, indicating the presence of the first harmonic of the sunspot cycle (not eliminated by the 11-year running window averaging), also show up on the plots in Figure 2. These variations are more pronounced in case of 
Table 1. Solar-interplanetary-magnetosphere parameters

\begin{tabular}{|c|c|c|}
\hline $\begin{array}{l}\text { Measured } \\
\text { data }\end{array}$ & Time range & Source \\
\hline$B$ & $1964-2007$ & http://omniweb.gsfc.nasa.gov/from/dx1.html \\
\hline$V$ & $1964-2007$ & http://omniweb.gsfc.nasa.gov/from/dx1.html \\
\hline$N$ & $1964-2007$ & http://omniweb.gsfc.nasa.gov/from/dx1.html \\
\hline TSI & $1964-2007$ & ftp://ftp.ngdc.noaa.gov/STP/SOLAR DATA/SOLAR IRRADIANCE \\
\hline$A a$ & $1868-2007$ & http://isgi.cetp.ipsl.fr/lesdonne.htm \\
\hline$I D V$ & $1872-2006$ & Svalgaard \& Cliver (2005, Table 3) \\
\hline Dst & $1957-2007$ & http://wdc.kugi.kyoto-u.ac.jp/wde/Sec3.html \\
\hline$A E$ & 1975-2007 & http://wdc.kugi.kyoto-u.ac.jp/wdc/Sec3.html \\
\hline$P C$ & $1975-2007$ & http://web.dmi.dk/projects/wdec1/pen/pen.html \\
\hline$C R$ & 1953-2006 & ftp://ftp.ngdc.noaa.gov/STP/SOLAR_DATA/COSMIC_RAYS/climax.tab \\
\hline$R$ & $1700-2007$ & ftp://ftp.ngdc.noaa.gov/STP/SOLAR_DATA/SUNSPOT_NUMBERS/INTERNATIONAL/yearly/YEARLY \\
\hline \multicolumn{3}{|c|}{ Reconstructed data } \\
\hline$B$ & $1872-2007$ & Svalgaard \& Cliver (2005), Rouillard et al. (2007), Demetrescu et al. (2010) \\
\hline$V$ & $1890-2007$ & Svalgaard \& Cliver (2005), Rouillard et al. (2007), Demetrescu et al. (2010) \\
\hline TSI & $1700-2007$ & $\begin{array}{l}\text { http://www1.ncdc.noaa.gov/pub/data/paleo/climate_forcing/solar_variability/lean2000_irradiance.txt; http:// } \\
\text { www.mps.mpg.de/projects/sun-climate/data/tsi 1700.txt (Lean et al. 1995; Lean 2000; Krivova et al. 2007) }\end{array}$ \\
\hline Fs & $1710-2007$ & Usoskin et al. (2002, 2007), Rouillard et al. (2007) \\
\hline$\Phi$ & $1710-2007$ & Usoskin et al. $(2002,2007)$ \\
\hline$C R$ & $1710-2005$ & Usoskin et al. (2002, 2007) \\
\hline
\end{tabular}

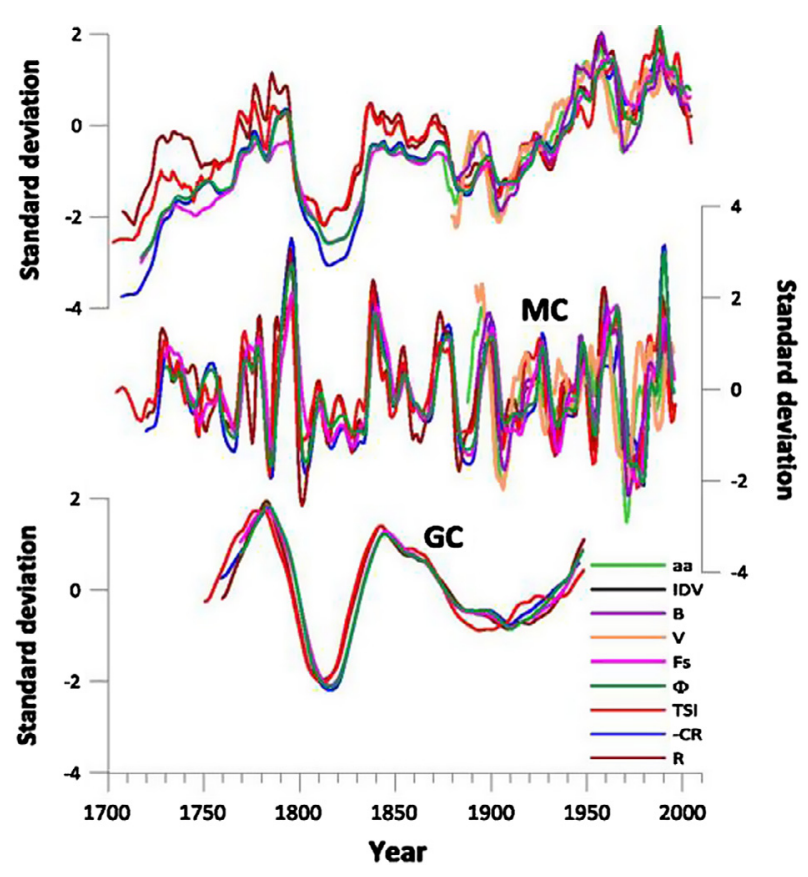

Fig. 2. Magnetic (Hale) cycle (MC) and Gleissberg cycle (GC) signals in the data analysed (middle and bottom panels, respectively), compared with the 11-year running averages (top panel), standardised for the common time intervals 1888-1991 and 17691948, respectively (from Demetrescu et al. 2010).

the solar wind velocity, illustrating a higher variability of $V$ in the ecliptic as compared to other parameters, in particular $B$ (see a more detailed discussion in Demetrescu et al. 2010).

The reconstructions based on data from cosmogenic isotope ${ }^{10} \mathrm{Be}$ in ice cores (Caballero-Lopez et al. 2004; McCracken 2007; Steinhilber et al. 2010) allowed to retrieve indirect information to confirm our findings, namely information regarding the long-term evolution of the reconstructed solar outputs such as the open solar flux and the strength of the IMF at $1 \mathrm{AU}$ that we have compared above with the geomagnetic response. Within limits imposed by the time sampling of data points (annual means and/or 22-, 40- and 25-year running averages), reconstructions based on ${ }^{10} \mathrm{Be}$ data agree well with reconstructions used by us in deriving our conclusions.

\section{Response of the terrestrial environment to solar activity}

In addition to the long-term solar activity, described in the previous section, the Sun exhibits many different signatures of short-term activity such as solar flares and prominence/filament eruptions. However, the eruptive phenomenon responsible for major space weather effects at the Earth is the CME. In CMEs, a great amount of matter and magnetic field is released, moving with speeds of several hundred (or thousand) kilometres per second. As a result, ICMEs, as counterparts of CMEs in the interplanetary medium, constitute large transient disturbances of solar wind and IMF that may impinge on the Earth's magnetosphere, thereby initiating a geomagnetic storm, whose effects are detectable over the whole magnetosphere-ionosphere-thermosphere system.

Active regions and quiescent filament regions have closed magnetic field structure meanwhile coronal holes have open magnetic field structure. High-speed streams of solar wind from coronal holes are the main cause of geomagnetic disturbances in the period around solar minimum, although the intensity of these disturbances cannot be compared with the largest storms caused by CMEs (Borovsky \& Denton 2006; Turner et al. 2009). Coronal holes are stable formations that can survive over several solar rotations. Stream interaction regions (SIRs) can thus periodically pass over the Earth causing recurrent geomagnetic storms. It makes forecast of such disturbances much easier (Bochníček \& Hejda 2002) than in the case of eruptive phenomena.

The standard model for the development of an eruptive flare driven by a rising loop involves the expansion of closed coronal 
magnetic fields. As this flux rope moves upwards, an inflow occurs behind it, driven by external magnetic pressure. The upward flow creates elongated field lines with opposed orientation to form a current sheet and ultimately to reconnect. This reconnection then explains the formation of the arcade of flare loops (Pick et al. 2006). The evidence of reconnection can be found in a broad spectrum of observations (Schwenn 2006); $\mathrm{X}$-ray and radio bursts will be mentioned here.

Hard X-rays are able to properly reveal particle acceleration and energy release in the low corona. There is a broad range of coronal sources including the footpoint sources of the flare impulsive phase. All of these sources require particle acceleration to high energies, and the particle acceleration produces a hard X-ray signature characteristic of CME sources. The signatures of solar particle acceleration are sometimes accompanied by SEP events.

Hectometric-kilometric Type II radio bursts are generated by energetic electrons, are long lasted and they decrease in frequency with time. The rate of the downward drift in frequency for Type II is consistent with a shock moving through the corona and solar wind (Nelson \& Melrose 1985) and they have been directly associated with interplanetary shocks observed with in situ spacecraft (Cane et al. 1982; Reiner et al. 1998). On the other hand, Type IV radio bursts are generated by energetic electrons that might come from the continued reconnection occurring beneath the CME (Gopalswamy 2011), so they may be indicative of material moving away from the corona (Kosugi \& Shibata 1997). From a statistical study, Gopalswamy (2011) found that this type of radio burst is associated with very energetic CMEs (average speed $\sim 1200 \mathrm{~km} \mathrm{~s}^{-1}$ ). So solar radio bursts provide important diagnostics of the solar eruption and the ambient medium through which the solar disturbances propagate and generate geomagnetic response.

Solar missions are currently gathering data with unprecedented resolution, not only in space and time, but also in wavelength. Therefore, new opportunities to improve the scientific knowledge on the triggers of geomagnetic disturbances arise, leading to develop new forecasting tools based on solar observations. These solar-based tools (see e.g., Kim et al. 2005; Gleisner \& Watermann 2006a, 2006b; Robbrecht \& Berghmans 2006) are able to forecast in advance - one to three days depending on the solar wind speed - to those prediction schemes based on the knowledge of interplanetary parameters (see e.g., Boberg et al. 2000; Gleisner \& Lundstedt, 2001a, 2001b; Lundstedt et al. 2002a, 2002b). For practical applications the first can serve as a preliminary warning, which is then confirmed or cancelled by the latter. In both cases, solar and interplanetary data based, the prediction can be obtained from physical laws (mainly based on empirical approaches) or by using mathematical models such as neural networks.

An Artificial Neural Network (ANN) is inspired by the functional aspects of biological neural networks. An ANN consists of an interconnected group of artificial neurons that changes its structure based on external or internal information that flows through the network during the learning phase. The neural-network learning phase is the process during which the sets of input data together with the corresponding desired output data are presented in successive steps to the ANN; the strengths of the inter-neuron connections are adjusted through an algorithm (e.g., back-propagation algorithm, based on the backward propagation of errors). The process is repeated in an iterative loop. The aim of the learning is setting up the connections in order to have the neural-network outputs near the observed data. Specifically, with a view to forecasting geomagnetic disturbances (outputs) driven by CMEs by means of an ANN, solar observations as input data (inputs) are needed.

Although huge amount of observations of CME phenomena have been collected in the last two decades, not only through coronagraphs but also through radio observations, these data are rather inhomogeneous and uneasy to be parameterised. On the other hand, the parameters of solar energetic events are published daily in tabular form by NOAA, Space Weather Prediction Center, Boulder, and the time series are complete and homogeneous since 1996 (http://www.swpc.noaa.gov/ $\mathrm{ftpmenu} /$ warehouse.html). That is the reason why these data have been used as a proxy of CMEs in the primary statistical evaluation, since major CMEs exceptionally occur without major flaring are only few cases compared to the long homogeneous data series.

\subsection{Analysis of the geoeffectiveness of solar energetic events}

Bochníček et al. (2007) performed an analysis based on data of solar energetic events, published in 1996-2004 by the USAF/ NOAA in the form of daily reports. X-ray flares (denoted in the reports as XRAs) and solar radio bursts (denoted as RSPs) were considered. The geoeffectiveness of the individual events was classified into three categories according to the intensity of the geomagnetic response, expressed by the geomagnetic $K p$ index. A disturbance was considered strong $(s)$ (medium, $m$ ) if the $K p$ index reached a value of $6(5)$ at least three times over the course of the response (no longer than $36 \mathrm{~h}$ ). The disturbance was considered weak $(w)$ if the $K p$ index reached a value of 5 at least once during the response, followed by no fewer than twice the value of 4 . In all other cases the response was considered insignificant.

The key task of this study was to coordinate the geomagnetic disturbances with the corresponding solar energetic events. Analogously to Wang \& Wang (2006) we used a fixed 30-120 h backward-time window to look for a candidate for the geomagnetic disturbance. As it has been mentioned above, one of the causes of enhanced geomagnetic activity may also be SIRs emanating from coronal holes, which are not a subject of this study. When analysing complicated situations (about 10\% of all cases), we drew particularly on the interplanetary parameters measured by the ACE satellite at libration point L1. To determine the actual solar source as well as the approximate time which has lapsed from the occurrence of the event on the Sun, we inspected the behaviour of the IMF and the solar wind parameters: solar wind velocity $\left(V_{\mathrm{sw}}\right)$, proton density and kinetic proton temperature $\left(T_{\mathrm{p}}\right)$, which, obtained as the corresponding velocity moment of the distribution function (see, e.g., Baumjohann \& Treumann 1997), should not be interpreted in the thermodynamic sense but as a measure of the spread of the particle distribution in velocity space. For example, SIRs can be easily recognised by a concurrent temperature rise and density drop on the leading edge of a high-speed stream (Crooker et al. 1999), while in situ signatures of CMEs include observed proton temperature lower than the "expected" $T_{\mathrm{p}}$ determined from the typical $V_{\mathrm{sw}}-T_{\mathrm{p}}$ correlation, which usually appears following interplanetary shocks. Although a shock is not an in situ signature of the CME itself however it is an often used and reasonably well-understood signature associated with many CMEs (Zurbuchen \& Richardson 2006).

Analysis of the particular energetic event types indicates that the degree of their geoeffectiveness depends on their size 
and on their solar-disc location. The mere information that a solar XRA event has occurred on the solar disc is insufficient to produce a reliable forecast of geomagnetic disturbances. XRAs of classes B and C were geoeffective in only 2\%, XRAs of class $\mathrm{M}$ in $8 \%$ and XRAs of class $\mathrm{X}$ in $33 \%$ of cases. The probability of having a geomagnetic response increases dramatically if the XRAs are associated with metric Type II and Type IV RSPs (Schwenn 2006). X-ray flares, which occurred in the central region defined by heliographic coordinates $\left[30^{\circ} \mathrm{E}\right.$, $\left.30^{\circ} \mathrm{W}\right]$ and $\left[30^{\circ} \mathrm{S}, 30^{\circ} \mathrm{N}\right]$, will very likely indicate a geomagnetic disturbance not only for X- but also for M-class XRAs (Bochníček et al. 2007).

\subsection{Application of Artificial Neural Networks}

In constructing the forecasting scheme, we first determined the relation between the flare characteristics (flare class, RSP type and location on the solar disc) and the probability that the degree of its geoeffectiveness is at least $w$. The solar disc was divided into areas of 18 degrees in heliographic latitude and longitude. In each of these areas was calculated the ratio of geoeffective XRAs to the total number of XRAs which had occurred in that area. Since the analysis proved that XRA events occurring at high heliographic latitudes were rarely geoeffective, areas with heliographic latitudes over $45^{\circ}$ were assigned zero geomagnetic response probability. The same zero probability was assigned to areas located in the immediate vicinity of both East and West edges of the solar disc. The fact that these areas were not of the same size due to projection was taken into account.

The classic backward propagation algorithm was used to realise the numerical training. To guarantee the stability of the results, nine neural networks were trained independently, with their median being considered final.

Figure 3 shows the distribution of geoeffective areas on the solar disc for the different XRA classes, and the different types of accompanying RSPs as well. These geoeffective areas display a moderate asymmetry with respect to the solar equator and the central meridian. The time series are too short for reliable conclusions but seems that the central meridian asymmetry relates to the Sun's rotation, and the cause of the equatorial asymmetry might result from the polarity of the solar magnetic field. It should, therefore, be interesting to monitor this phenomenon during the 24th solar cycle. From a long-term point of view, the parity of the cycle could prove to be an important parameter for the neural network. Unfortunately, because of the prolonged solar minimum the data obtained are only sufficient to yield preliminary results.

The second step of the forecasting scheme was to determine the intensity of the geomagnetic response associated with the geoeffective flares. The drawback of the forecast based on a three-stage scale ( $w, m$ and $s$ ) is the excessively high value of some non-diagonal terms of the ANN matrix. However, if the table is reduced to $2 \times 2$ cells by combining $w$ and $m$, the diagonal terms become dominant (see Tables 5 and 6 of Valach et al. 2007). In other words, the model can be used satisfactorily to forecast whether or not the expected response will be severe. The forecasting scheme was trained using events observed in the years 1996-2004 and tested using data from the time interval 2005-2006. For instance, geomagnetic response was correctly forecast after the X-ray flares of Type B, C, M or X accompanied by the radio bursts of Types II and IV in $56 \%$ of cases; altogether 41 such responses were observed, of which 23 were forecast correctly; at the same time 7 false alarms were produced. The forecast improves with increasing XRA class. For class X, 13 out of 15 observed responses (87\%) were forecast correctly and 2 false alarms were issued. In addition to statistical assessment of the results, the full list of events was published, along with a comparison of the observations to the forecasts (Valach et al. 2007).

The high percentage of successful forecasts of geomagnetic disturbances confirms the close connection between solar flares and CMEs (Schwenn 2006).

Gleisner \& Watermann (2006a) found that enhancement of the $\geq 10 \mathrm{MeV}$ high-energy proton flux (HEPF) close to CME onset can be used to indicate whether CMEs approaching Earth will be followed by a severe geomagnetic disturbance. Ranking the CMEs by velocity and SEP-flux enhancement shows that the latter indicator results in better discrimination between highly geoeffective CMEs and those less geoeffective.

Taking into account the previous result, in the next stage we tested whether the success rate of the neural network's prediction scheme can be improved by including additional information about the HEPF. The increase of HEPF to $>10 \mathrm{MeV}$ was characterised in two ways:

1. The increase was shown by the quantity $\Delta \log F=\log$ $\left(F^{\mathrm{post}} / F^{\mathrm{pre}}\right)$, where $F^{\mathrm{pre}}$ is the minimum value of the SEP flux during the 6 hours prior to XRA occurrence and $F^{\text {post }}$ is the maximum value of the SEP flux during the $12 \mathrm{~h}$ after the XRA. This measure of HEPF enhancement to $>10 \mathrm{MeV}$ is roughly the same as that used by Gleisner \& Waterman (2006a).

2. To eliminate impulsive HEPF events from the deliberation, which are not produced by CME-driven shocks (as opposed to gradual events), the adopted measure of HEPF was slightly modified to $\Delta \log \Phi=\log \left(\Phi^{\text {post }} / F^{\text {pre }}\right)$, where $\Phi^{\text {post }}$ is the maximum value of the HEPF during the $10 \mathrm{~h}$ following the $12 \mathrm{~h}$ after XRA occurrence.

The success of the use of these parameters is shown in Table 2 by the contingency coefficient $C$, which is defined as $C=\left[\chi^{2} /\left(\chi^{2}+N\right)\right]^{1 / 2}$, where $\chi^{2}$ is a quantity known from the statistics of discrete characters and $N$ is the number of samples in the statistical set. The values of quantity $C$ range from 0 to 1 , and the higher the value of $C$, the more successful the forecast has been.

\section{Response of the terrestrial environment to the solar wind}

In the task of forecasting geomagnetic disturbances, solar inputs are preferred over solar wind inputs, since they could provide warning results sooner. However, at present, forecasting magnetospheric responses based on solar observations is not accurate enough for practical purposes.

A prediction scheme joining the edges of the Sun-to-Earth chain, not becoming aware of middle stages, is based on statistical studies of events with a truthful cause-effect relationship. However, there are exceptional events with a significant disturbance whose statistical weight usually is very low and therefore to extrapolate results about geoeffectiveness of these events only on the base of solar observations is a risky action. The paper by Rodriguez et al. (2009) is an example of how inaccurate may be the result of doing this extrapolation in the case of assuming that the closer is the solar source of a CME to the central meridian, the larger disturbances are expected. On the other 


\section{E. Saiz et al.: Geomagnetic response to solar activity}

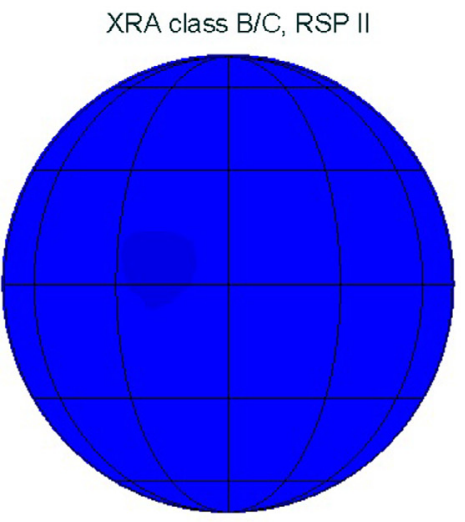

XRA class $M$, RSP I

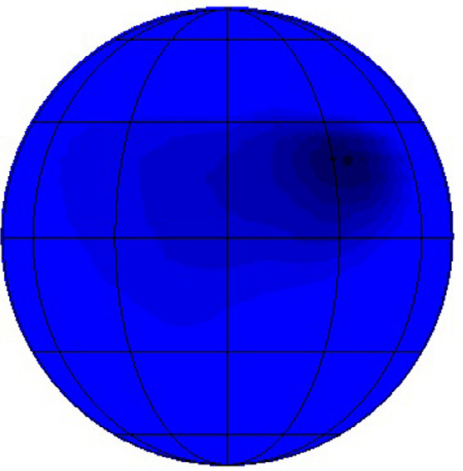

XRA class X, RSP II

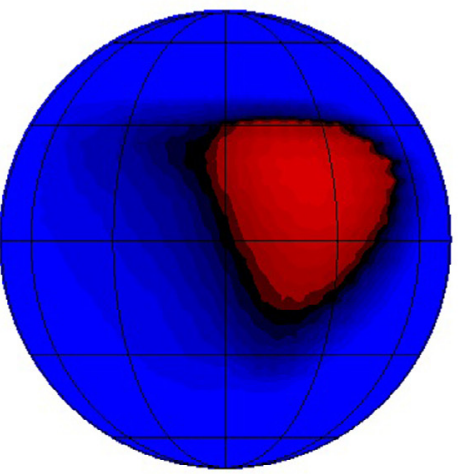

XRA class B/C, RSP I\&IV

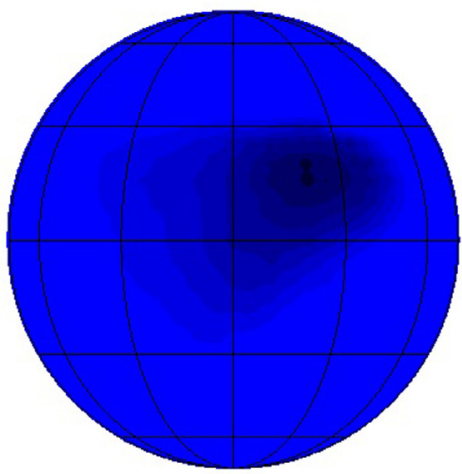

XRA class M, RSP $\| \& I V$

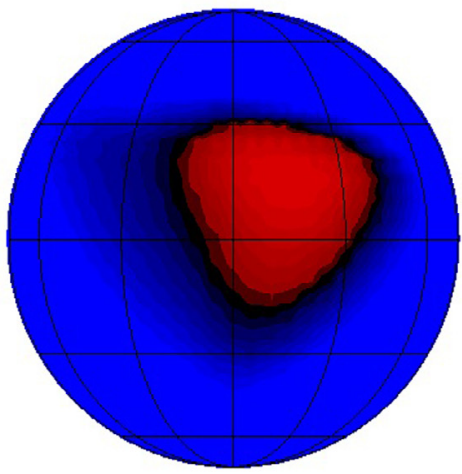

XRA class X, RSP \|\&IV

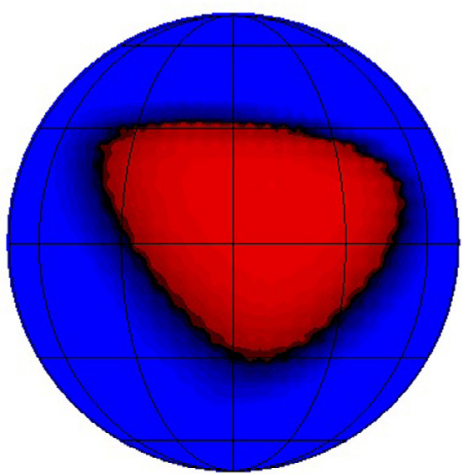

XRA class B/C, RSP IV

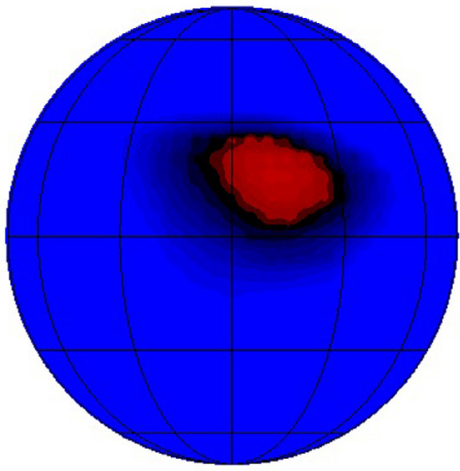

XRA class M, RSP IV

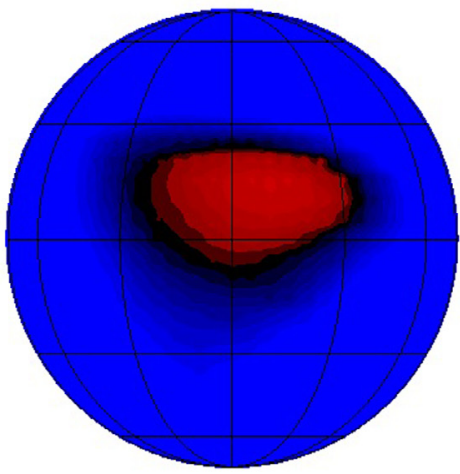

XRA class X, RSP IV
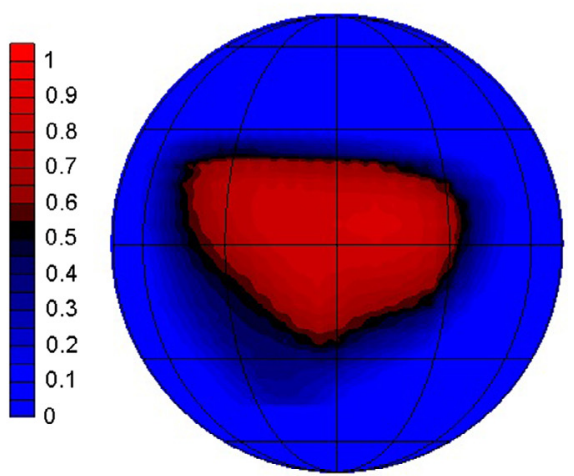

Fig. 3. Distribution of the probability that the solar events released on a given place on the solar disc produce a geomagnetic response (at least $w$ ). The areas in which the probability is greater than $50 \%$ are shown in red (from Valach et al. 2007).

Table 2. Success rate of forecasting geomagnetic response in terms of Cramer's $V$ and the contingency coefficient $C$ for independent test specimens in 2005 and 2006 (from Valach et al. 2009).

\begin{tabular}{lc}
\hline \hline Input parameters & Contingency coefficient \\
& $C$ \\
\hline$\lambda, \varphi$, RSP II/IV, XRA class & 0.467 \\
$\lambda, \varphi$, RSP II/IV, XRA class, $\Delta \log (F)$ & 0.553 \\
$\lambda, \varphi, \Delta \log (\Phi)$ & 0.544 \\
$\lambda, \varphi$, RSP II/IV, XRA class, $\Delta \log (\Phi)$ & 0.637 \\
\hline
\end{tabular}

hand, these events out of the statistics, which are usually cause of the most severe disturbances, offer an extraordinary opportunity to go further in the knowledge of heliospheric physics and, as a result, on acquiring better results on the forecasting process.

The challenge to predict accurately and as soon as possible, that is, from solar observations and based on the knowledge of physical processes, requires an expertise on all the stages from the Sun to the Earth of the phenomena. In this scenario, interplanetary signatures are the key to go forward towards the Earth (linking interplanetary and magnetospheric observations) and backwards to the Sun (linking interplanetary and solar observations) to finally discover accurately the trigger of the observed disturbance. Dasso et al. (2008), Rodriguez et al. (2009) and Cid et al. (2012) are some examples of detailed studies of selected events that evidence that the analysis of interplanetary signatures is a key element to fully understand the event. They also show the difficulty in the identification of the solar trigger and sometimes even the uncertainty in the selected candidate. 
Due to its significance, solar wind data are preferred to solar data as inputs for many space weather products, which provide good results for typical disturbances. However, for severe disturbances, the small number of historic events recorded renders the task of developing forecasting models based on statistical approaches hard, and therefore a better understanding of each part of the Sun-Earth chain is needed.

In the following, we show our most significant achievements in different issues, all related to the link between the interplanetary medium and magnetosphere.

\subsection{Enhanced geoeffectiveness caused by large changes in solar wind parameters}

Cerrato et al. (2011) searched for solar sources and related interplanetary structures that could have been associated with the seven largest Dst-index decreases during $1 \mathrm{~h} \quad(\Delta D s t$ $<-100 \mathrm{nT}$ ) throughout solar cycle 23 . The seven events were triggered by interplanetary signatures that arose as a consequence of interactions among different solar ejections. The interactions arose at different stages of the scenario from the Sun to the interplanetary medium: one took place at the solar surface between segments of a filament; others occurred in the interplanetary medium, revealing characteristics of ejecta or multimagnetic clouds (MultiMCs). In other cases, shock waves overtook or compressed previous interplanetary CMEs (ICMEs) and, at other times, interactions also appeared between magnetic clouds (MCs) and streams. As for interplanetary-medium signatures, all events presented a large change to southward direction of the $B_{z}$ component of the IMF, frequently associated with a compression process and density enhancements.

Weigel (2010) showed that the solar wind density can significantly enhance the storm intensity. The solar wind density influence was quantified by studying the response of the ring current, as indicated by Dst index, to the solar wind electric field. Two statistical approaches were considered: (1) dataderived impulse response function and (2) the relationship between the integrated value of $D s t$ to the integrated value of the interplanetary electric field during geomagnetic storm intervals. Both approaches indicate that density modifies the ability of a given value of electric field to create a Dst disturbance.

Moreover Du et al. (2008) analysed the magnetic storm that occurred on 21-22 January 2005 which can be considered highly anomalous because the storm main phase is developed during northward IMF $B_{z}$. They pointed out that a non-compressive density enhancement could play a key role in the large response of the ring current. They proposed that there is first energy storage in the magnetotail and then, when IMF was northward, a delayed energy injection in the ring current. The storage may arise from intense dynamic pressure by shock and discontinuity impacting the magnetosphere.

Lopez et al. (2004) also pointed out density enhancements as causing large Dst decreases. During periods of strongly southward IMF, such as that which occurs in the main phase of a storm, the compression ratio of the low Mach number shock is strongly affected by the variations in solar wind density. They found that higher solar wind densities lead to a larger compression ratio across the shock, which produces larger magnetosheath fields. Since it is the magnetosheath that is actually in contact with the magnetopause, the stronger magnetosheath field applied to the magnetopause results in an increased polar cap potential - driven by the dayside reconnection rate - and a higher level of dissipation. However Lopez et al. (2004) noted that it is a question of the amplification of the solar wind magnetic field by the bow shock rather an issue strictly of the solar wind kinetic energy flux.

Large changes to southward direction of the $B_{z}$ component of the IMF had already been related to large variations in Dst by Saiz et al. (2008). Their results for a sample covering data obtained over a period of 10 years showed that there might be energy transfer from the solar wind to the magnetosphere, not only because of the arrival of a southern IMF at the nose of the magnetopause and through reconnection leading to a subsequent large-scale convection flow towards the tail, but also because of fluctuations in the $B_{z}$ component of the IMF. These results indicate that more contributions than just that from the dawn-dusk convective electric field could be involved in the injection function from the solar wind to the magnetosphere.

\subsection{Forward steps in the energy balance between the solar wind and the ring current}

As a consequence of the important process of magnetic reconnection which takes place in the magnetosphere at both the dayside magnetopause and the magnetotail (Dungey 1961), energy transfer from the solar wind to the magnetosphere occurs and its effects can be measured at the terrestrial surface using various geomagnetic indices.

Although many other geomagnetic indices are also used as proxies of terrestrial disturbances, the Dst index is one of the most extensively used tracers because of its clear physical meaning: it represents the total kinetic energy in the ring current plasma (Dessler \& Parker 1959; Scopke 1966). The prediction equation for Dst was introduced by Burton et al. (1975),

$$
\mathrm{d} D s t^{*} / \mathrm{d} t=Q-D s t^{*} / \tau,
$$

where $D s t^{*}$ is the $D s t$ index corrected for the contributions from magnetopause currents, $Q$ is the source term and the last term is the ring current loss function, which is controlled by the decay time, $\tau$.

This equation determines the evolution of $D s t$ entirely from conservation of energy: energy supply and loss rates are taken into account, although the processes by which energy is transferred to the ring current plasma are not considered. More recently, numerical simulation codes have been developed and applied to predict Dst (e.g., Liemohn et al. 2001; Jordanova et al. 2003). The models describe in detail the energising and loss processes, but do not impose or even consider conservation of energy the electric and magnetic fields are stipulated, without any requirement of consistency with plasma, so changes in the energy content in the magnetic field are not taken into account. The different approaches to the problem of the evolution of Dst (empirical or based on the physical processes involved, by numerical simulations, or by analytical solution) are evidence for complementary steps towards a full self-consistent description of the ring current still to be achieved.

\subsubsection{About the injection function}

The fact that predictions of Dst based on both methods (energy balance and physical processes) agree reasonably well with one another and with observations caused Vasyliunas (2006) to reexamine the Burton-McPherron-Russell (BMR) equation used for predicting Dst. He found that the injection function for the plasma energy content is not a unique source term, but magnetotail contributions arising from the addition of open flux by dayside reconnection and from its removal by nightside reconnection are also important. The assumption that the empirical 
E. Saiz et al.: Geomagnetic response to solar activity
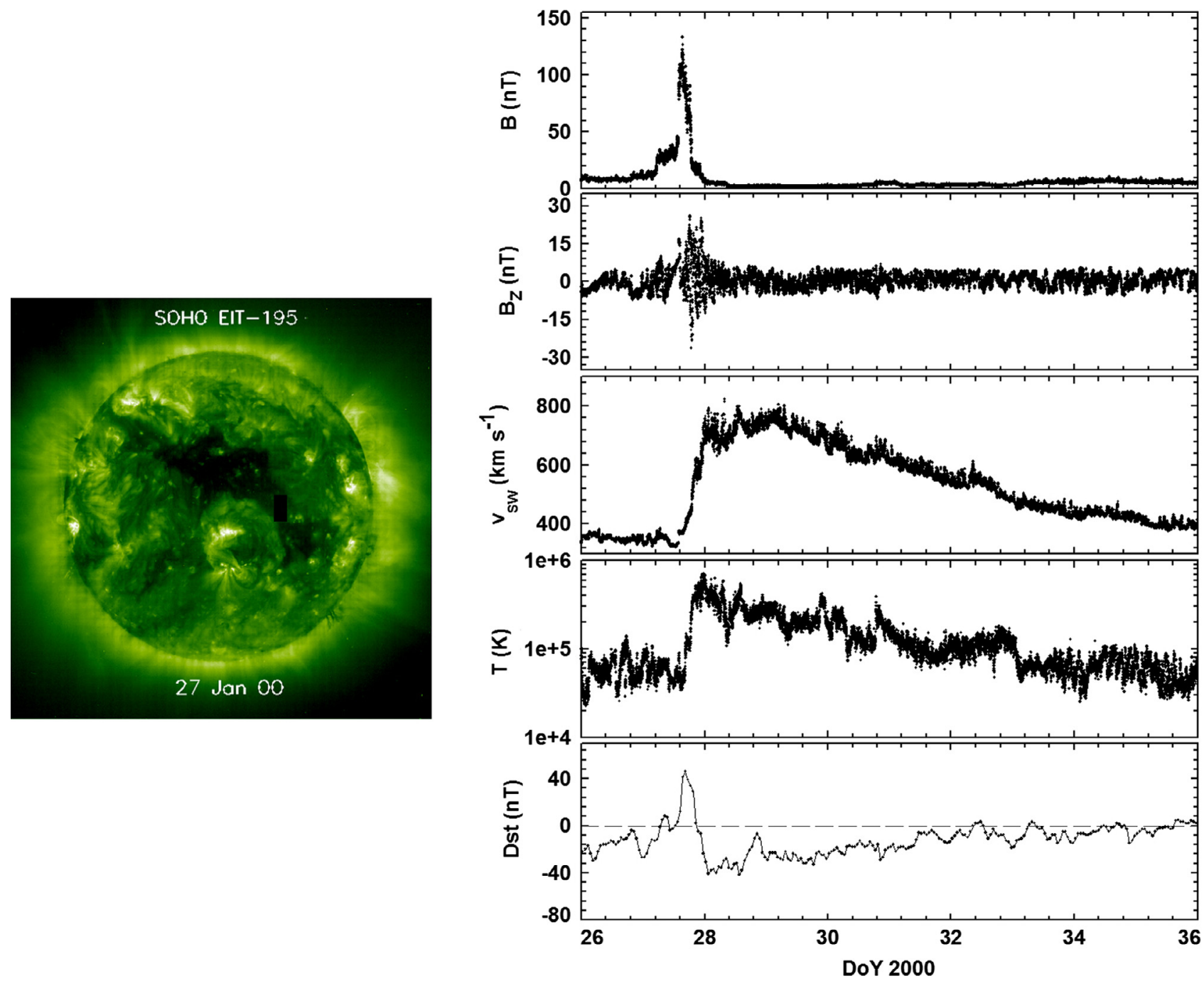

Fig. 4. (Left) SOHO-EIT $195 \AA$ Amage from 27 January 2000, showing a huge coronal hole. (Right) Interplanetary parameters from the ACE spacecraft (from top to bottom: magnetic field strength and $z$-GSM component, and solar wind velocity and temperature) and $D s t$ index from Kyoto showing the fast stream from the coronal hole shown in the EIT image. The Dst index remains disturbed for more than 5 days.

source function for Dst directly represents energy injection into the ring current plasma is, therefore, not valid unless the open magnetic flux does not change, which holds only for averages over sufficiently long timescales. However, short timescales are most relevant for large variations in the terrestrial magnetic field (and, therefore, for Dst and indices constructed from the geomagnetic field). Therefore, improving our understanding of such short timescales is absolutely necessary because Geomagnetic Induced Currents (GICs) events, for example, are directly related to large variations in the geomagnetic field. Besides firm theoretical reasons for this statement, a strong linear correlation between the time-rate-of-change of the magnetic field and measured GICs has been observed at all latitudes (Thomson et al. 2010). In the past GICs have been widely reported and analysed in Canada, Finland and Scandinavia, and therefore there is a perception that GICs are a risk only for power grids at relatively high latitudes (the most known example is the collapse of the Hydro Quebec power system on 13th March 1989). However, GIC impacts have been reported in more mid-latitude countries such as the UK, Japan and USA, or in South Africa, China and Brazil at low latitudes (e.g., failure in a large South African generator transformer three weeks after the Halloween storm of October 2003) (see references in Thomson et al. 2010).
As the magnetospheric and ionospheric currents that drive GICs are different at different latitudes, this means that at higher latitudes the auroral electrojet disturbances will induce largely electric fields, but at mid- and low-latitude ring current and equatorial electrojet will play a major role. Accurate prediction of GIC risk then requires accurate prediction of changes in the geomagnetic field at all latitudes. In this study we focused on changes in the Dst index, which is related to mid-low latitudes.

In some cases the Dst index can remain below its quiet-day value for days, even in the absence of a storm (see Fig. 4). Such anomalous behaviour of the Dst recovery is observed during times exhibiting continuous auroral activity, referred to as High Intensity Long Duration Continuous AE Activity (HILDCAA; Tsurutani \& Gonzalez 1987; Søraas et al. 2004). It has been determined that this kind of event is associated with low-level injection of protons into the outer portion of the ring current, which is related to fluctuations in the solar wind magnetic field, where the varying $B_{z}$ component causes intermittent reconnection and sporadic injection of plasma-sheet energy into the ring current, thus prolonging its final decay. However, no term in the BMR equation considers that the Dst decrease in HILDCAAs is related to fluctuations in $B_{z}$, since the duration of the southward $B_{z}$ component is not long enough to explain the phenomenon. 
Cid et al. (2013) have undertaken a study to check the cause-effect relationship during the main phase of the storm between duskward interplanetary electric fields, $E_{y}$ and the decrease of the $S Y M-H$ index which, with 1-min resolution, can be regarded as a high resolution Dst index (Wanliss \& Showalter 2006). Their results suggest that an injection function which depends only on $E_{y}$ cannot explain the energy released from the solar wind to the terrestrial magnetosphere for timescales shorter than $8 \mathrm{~h}$. Moreover, they show that there is some missing energy in the energy-balance equation between the interplanetary medium and the magnetosphere for those events where $S Y M-H$ decreases fast $(-100 \mathrm{nT}$ in $<8 \mathrm{~h})$. The missing energy term from these empirical results might be related to the open magnetic flux proposed by Vasyliunas (2006). However, more effort is needed to check whether this hypothesis is correct.

\subsubsection{About the recovery phase}

After the ring current particles are energised as a consequence of the energy input from the solar wind, which corresponds to a decrease in the Dst index or main phase of the storm, a variety of loss processes such as charge exchange, Coulomb interactions and wave-particle interactions (Jordanova et al. 1996; Kozyra et al. 1997, 1998) begin to be relevant agents driving the storm. A subsequent stage of magnetospheric evolution starts: the ring current decay, or recovery phase, which is observed as a slower recovery period in which Dst gradually returns to its quiet-time value (Takahashi et al. 1990; Kozyra et al. 1998; Feldstein \& Dremukhina 2000).

The decay time of the ring current is an important parameter to be known, because the particle injection rate cannot be determined without sufficient knowledge of the decay parameter. It has been observed that the Dst decay following a geomagnetic storm shows a two-phase pattern, including an early fast recovery followed by a slower phase. Many theories have been proposed to explain the observations. It has been proposed that differential decay rates of different ion species may lead to the two-phase decay, as explained in the review paper of Daglis et al. (1999). However, Jordanova et al. (2003) and Kozyra \& Liemohn (2003) proposed a changeover from rapid removal by the decreased convection electric field and an outflow through the magnetopause on the dayside during the main and early recovery phases to much slower charge-exchange removal of trapped ring current particles during the late recovery phase. Later, Liemohn \& Kozyra (2005) supported these arguments based on idealised simulations.

From another point of view, a number of studies have been devoted to find decay time values by either considering the recovery phase of the storm as a single, two or even more periods (Burton et al. 1975; Hamilton et al. 1988; Ebihara et al. 1998; Dasso et al. 2002; Kozyra et al. 2002; Weygand \& McPherron 2006; Monreal MacMahon \& Llop 2008). Among these studies, some considered a constant recovery time (e.g., Burton et al. 1975), others depended on the convective electric field $E_{y}$ (O'Brien \& McPherron 2000) or also on the dynamic pressure (Wang et al. 2003).

More recently, Aguado et al. (2010) proposed a hyperbolic decay function to model the entire recovery phase of intense geomagnetic storms $(D s t<-100 \mathrm{nT})$,

$$
\operatorname{Dst}(t)=\frac{D s t_{0}}{1+\frac{t}{\tau}},
$$

where $D s t_{0}$ and $\tau$ represent the $D s t$ value at $t=0$ (the time when the recovery phase begins) and the characteristic recovery time of the ring current, respectively.

They considered all intense storms in the period 1963-2003 which did not exhibit a significant injection of energy during the recovery phase, and applied a superposed epoch method to determine the average recovery phase at several intensity ranges, from -100 to $-400 \mathrm{nT}$, to constrain the possible dependence of the recovery time on the intensity of the storm. This kind of function not only fits experimental data with higher correlation factors than the exponential relation at every intensity range, but the function is also consistent with the diverse nature of the loss mechanisms involved with different lifetimes at different stages and different storm intensities, as described above. So, a unique function (but hyperbolic instead of exponential) allows reproduction of the global behaviour of the entire recovery phase of the storm, including the impulsivity shown in the early recovery phase, as observed especially for more intense geomagnetic storms.

From a physical point of view, the great difference in proposing an exponential or hyperbolic ring current decay is that the exponential decay is based on the assumption of a decay rate which is proportional to the energy content of the ring current (through the Dessler-Parker-Sckopke relationship), that is, on a linear dependence of $\mathrm{d} D s t / \mathrm{d} t$ on $D s t$. However, the hyperbolic decay assumes a quadratic dependence of these magnitudes. That provides evidence of a non-linear relationship between the Dst variation rate and $D s t$ itself. Therefore, the recovery phase of the magnetosphere at low latitudes, as described by Dst index, exhibits a non-linear behaviour.

Another notable result is that the ring current recovers on a characteristic timescale which depends on the intensity of the storm. In the range of intensities analysed, the parameters $D s t_{0}$ and $\tau$ showed a linear dependence. Therefore, it is possible to work out in advance how much time the ring current will need to recover during its quiet time, which is important for space weather purposes.

An important improvement of the model was introduced when some historic superstorms $(D s t<-250 \mathrm{nT})-$ the most extreme storms ever detected, such as the Carrington storm in 1859 - were considered with a dual purpose: to validate the hyperbolic model for the large range of Dst and to find out whether the linear dependence between $\tau$ and $D s t_{0}$ still holds. Cid et al. (2013) show the high accuracy of the hyperbolic fitting in reproducing the recovery phase of Dst index in extreme storms. Figure 5 shows, as an example, the results of the hyperbolic decay fitting to one of the extreme geomagnetic events: the large storm in July 1928 recorded at Alibag magnetometer.

As an additional point, the results of that study demonstrate that the time that takes the ring current to recover depends in an exponential way on the intensity of the storm. The exponential function obtained is consistent with the linear function proposed by Aguado et al. (2010) when the severity of the storm diminishes.

\subsection{High-latitude solar wind-magnetosphere-ionosphere coupling}

Many communication systems rely on the propagation of radio waves through the ionosphere. The varying characteristics of the ionosphere can cause significant disturbances in the radio signals (i.e., phase and amplitude scintillation) and cause errors or signal disruption in the respective services. Therefore, 


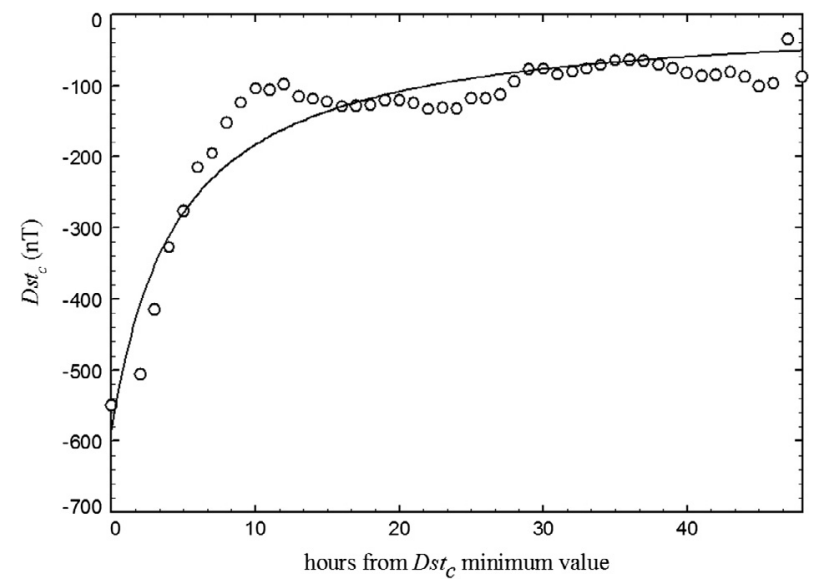

Fig. 5. The computed Dst, Dst $t_{c}$, as a function of time during the recovery phase of the large storm registered at Alibag in 1928. The line corresponds to the fitting results to a hyperbolic decay function.

forecasting ionospheric disturbances related to solar activity is a paramount aim for space weather because of their effects and their societal and economic impacts.

Besides radio bursts, interplanetary signatures and their interaction with the magnetosphere, and then the interaction between the magnetosphere and the ionosphere, should address the cause of these disturbances. On the other hand, since the ionosphere is a highly dynamic system it is also very important to characterise its state not only in storm-time conditions but also in quiet conditions.

The energy transfer from solar wind to magnetosphere is, from a global point of view, mainly controlled by the reconnection and magnetospheric convection processes. The first one allows the transport of energy and momentum into the magnetosphere by permitting solar wind to cross the magnetopause and once it is there to drive the second one. The transport of plasma across a magnetopause produces mixed plasma regimes adjacent to the interface that are known as boundary layers. Spacecraft observations have revealed that the low-latitude boundary layer (LLBL) earthward of the magnetopause contains a mixture of magnetospheric and magnetosheath plasma with a generally tailward bulk flow (see, e.g., the review by De Keyser et al. 2005). Observations have also revealed that although the layer is a quasi-permanent feature of the terrestrial magnetosphere, its properties are also highly variable (Masters et al. 2011 and references therein). To locate the boundary layers (the open-closed field line boundary) is significant for understanding the magnetospheric dynamics. However it is hard to distinguish the magnetic field topology inside the LLBL based on plasma population observations (Phan et al. 2005; Bogdanova et al. 2008) and controversy surrounds about the extent to which magnetic field lines are open or closed. Particle measurements in the ionosphere from satellite observations are needed but cannot be used as unambiguous discriminators between closed and open field lines on the dayside (Oksavik et al. 2000). Therefore, determining reliable proxies to identify the location of the open-closed field line boundary is very important for the study of magnetosphere-ionosphere coupling since it is crucial for making accurate ionospheric measurements of many magnetospheric processes such as the rate reconnection, the size of the polar cap, etc. In this line of work a lot of recent papers can be mentioned (e.g., Hosokawa et al. 2003; Wild et al. 2004; Chisham et al. 2005; Imber et al. 2013).
The dominance of the processes involved in the formation of LLBL depends on the IMF orientation: during southward IMF, reconnection is commonly observed at the low-latitude magnetopause, however when the IMF is strongly northward, reconnection at the low-latitude magnetopause is less efficient or absent (Phan et al. 2005) and Kelvin-Helmholtz instability (Hasegawa et al. 2004) or diffusive entry (e.g., Johnson \& Cheng 1997) processes have been suggested to play a dominant role. On the other hand, evidence for LLBL formed by reconnection at high latitudes in both northern and southern cusps during northward IMF conditions also has been reported (e.g., Fuselier et al. 2002; Twitty et al. 2004; Lavraud et al. 2006; Marcucci et al. 2008; Dunlop et al. 2009) and strongly depends on the polarity of IMF $B_{y}$ (Šafránková et al. 2007).

Dayside auroral phenomenology provides critical information for understanding the interactions of the interplanetary medium with the Earth's magnetosphere. Manifestations of these interactions appear in the ionosphere as optical emissions, precipitating particles, plasma convection, electric fields and field-aligned currents (FACs).

While precipitating particles enhance the conductivity of the ionospheric plasma, the convection produces a system of electric fields. Both cause strong electric currents, mainly in the auroral zone within the dynamo region (90-150 km altitude), which lead to Joule heating, plasma instabilities and observable changes of the geomagnetic field on the ground.

The so-called dynamo concept (see, e.g., Amory-Mazaudier 2008) relies on the fact that the motion of a plasma across a magnetic field induces an electric field which produces an electric current via Ohm's law. This current produces in turn a magnetic field which creates both electric field via Faraday's law and Lorentz force which reacts on the motion. The two main large-scale dynamos in the Earth's environment are the ionospheric dynamo and the solar wind-magnetosphere dynamo. It is well known that magnetosphere-ionosphere system reacts to solar wind electric field variability like a high-pass filter, allowing electric fields to penetrate (Vasyliunas 1972; Kelley 1989) even to the magnetic equator, with efficiencies depending on the orientation of IMF $B_{z}$ component (Kelley \& Retterer 2008). These electric fields have been identified as the direct prompt penetration electric field associated with magnetospheric convection and/or the ionospheric disturbance dynamo electric field, with a characteristic delayed development, driven by Joule heating at auroral latitudes. While the magnetospheric dynamo causes electrical currents to be driven between the magnetosphere and the high-latitude ionosphere along geomagnetic field lines, the ionospheric disturbance dynamo alters the global circulation pattern in the thermosphere and ionosphere (Fejer \& Scherliess 1997).

Large changes in ionospheric electron density can be produced by enhanced disturbance electric fields associated to geomagnetic storms (Foster \& Rich 1998; Tsurutani et al. 2004). Likewise, the large amount of energy dissipated at high latitudes during storms or substorms can generate travelling atmospheric disturbances in the thermosphere (e.g., Prölss 1993) which can be propagated to middle-low latitudes and even into the opposite hemisphere leading to ionospheric fluctuations at those latitudes.

On the other hand, the combined particle precipitation patterns shape the auroral oval, whose diameter depends on the amount of open magnetic flux within the polar cap. This open flux is related to the rate of opening lines at the magnetopause and magnetotail (via reconnection) and thus to geomagnetic 
activity (Lester et al. 2006). For determining the potential associated with magnetospheric dynamo, it is necessary to find the polar cap boundary (i.e., the open-closed field line boundary). Since the polar cap connects the magnetic field of the Earth to that of the solar wind, it is an ideal region to investigate how the solar wind drives the magnetosphere-ionosphere coupling. Key research in this field emphasises the need to understand the nature of the electrodynamic coupling between both regions, whose characteristics affect each other and therefore cannot be properly understood when are treated separately.

Particularly, in the following subsections we will focus on how to characterise magnetic activity at the polar cap ionosphere from solar wind measurements and to point out how important are small-scale FACs, which connect high-latitude ionospheric currents and the magnetosphere.

\subsubsection{The PC indices and their potential for space weather monitoring}

The two polar caps are the regions of the Earth that are in closest contact with the solar wind. Hence, the magnetic variations, caused by electric currents induced by the solar wind and flowing in the polar upper atmosphere, could be considered the most directly available ground-based measure of the solar wind intensity. The solar wind electric field is, in most cases, the dominant factor driving high-latitude magnetospheric electric field structures and related plasma convection processes. The convection electric field could be characterised by the so-called "merging" (or "reconnection", or "geoeffective") electric field was defined by Kan \& Lee (1979):

$$
E_{\mathrm{M}}=V_{\mathrm{SW}} B_{T} \sin ^{2}\left(\frac{\theta}{2}\right)
$$

where $V_{\mathrm{SW}}$ is the solar wind velocity, $B_{T}$ the IMF's transverse component, $B_{T}=\sqrt{B_{Y}^{2}+B_{Z}^{2}}$, and $\theta$ the IMF clock angle, $\tan (\theta)=\left|B_{Y}\right| / B_{Z}, 0 \leq \theta \leq \pi$.

The Polar Cap PC index (PCN, PCS: northern, southern $P C)$ is derived from magnetic measurements from observatories within the Polar Caps and provides useful characterisation of the state of the polar ionosphere, describing the ionospheric currents related to the transpolar plasma convection.

Basically, the $P C$ index is defined to be a proxy for the merging electric field by assuming that there is proportionality between the horizontal magnetic field variations in the central polar cap, $\Delta F$, and the merging electric field, $E_{\mathrm{M}}$, and that the magnetic variations related to $E_{\mathrm{M}}$ have a preferred direction with respect to the Sun-Earth direction. Thus, on a statistical basis from observations of $\Delta F$ and $E_{\mathrm{M}}$

$$
\Delta F_{\text {PROJ }}=\alpha E_{M}+\beta,
$$

where $\Delta F_{\mathrm{PROJ}}$ is the projection of the magnetic disturbance vector, $\Delta \boldsymbol{F}$, in the direction most sensitive to the merging electric field. The parameter $\alpha$ is the "slope" and the residual $\beta$ is the "intercept" parameter. To calibrate magnetic variations with respect to the merging (geoeffective) electric field, the $P C$ index is defined by the inverse relation to make it equivalent to $E_{\mathrm{M}}$ :

$$
P C=\left(\Delta F_{\text {PROJ }}-\beta\right) / \alpha\left(\sim E_{\mathrm{M}}\left[\mathrm{mV} \mathrm{m}^{-1}\right]\right) .
$$

Thus, the $P C$ index can be considered a proxy for the solar wind merging electric field. The $P C$ index concept is based on the formulation of Troshichev et al. (1988).
The development of a $P C$ index was recommended by the International Association of Geomagnetism and Aeronomy (IAGA) in 1999 and the index in its present formulation (Troshichev et al. 2006) will probably soon be adopted by the IAGA as an international standard index.

The $P C N$ index for the northern polar cap is based on data from the Danish geomagnetic observatory in Thule (Qaanaaq), Greenland, while the $P C S$ index for the southern polar cap is based on data from the Russian geomagnetic observatory in Vostok, Antarctica. Both the PCN and PCS indices have been calibrated with respect to the merging electric field $\left(E_{\mathrm{M}}\right)$. Accordingly, the two index series are also mutually equivalent in a statistical sense. However, differences may arise as the result of both different conditions in the two polar caps (e.g., different solar illumination) and different responses to forcing from the solar wind, as well as of different responses to substorms. A further possibility is the combination of the two index series into a single one (Stauning 2007). The problem with large negative $P C$ index values in the sunlit hemisphere during strong and northward IMF (i.e., NBZ conditions) supports the construction of a combined $P C$ index $(P C C)$ from non-negative values only. Accordingly,

$$
\begin{aligned}
P C C= & {[(P C N \text { if }>0 \text { or else zero })} \\
& +(P C S \text { if }>0 \text { or else zero })] / 2 .
\end{aligned}
$$

Like the $E_{\mathrm{M}}$ values, the $P C C$ index values are non-negative, even during NBZ conditions, and they exhibit a stronger correlation with $E_{\mathrm{M}}$ than either the $P C N$ or $P C S$ indices. In addition, as a combination of northern and southern polar cap conditions, the $P C C$ index is more representative of the global disturbance level than the individual $P C N$ or $P C S$ indices.

The $P C$ indices monitor the geoeffective energy input supplied by the solar wind. The solar wind conditions are, of course, best monitored by in situ observations such as those obtained by the ACE satellite located at the L1 position. However, these observations, in particular those of the solar wind velocity, density and temperature, are sometimes hampered by the strong solar proton radiation that often accompanies the stronger outbursts and could be seriously misleading. In these cases, the $P C$ index provides a useful source for confirmation or back-up replacement of in situ solar wind observations.

The effects of the energy input from the solar wind to the magnetosphere at high latitudes are also monitored by other parameters and indices, like the cross-polar cap potential, $\Phi$, the auroral electrojet indices, $A E, A L$ and $A U$, the thermosphere Joule heating parameter, $J H$, the Auroral Power index, $A P$, at mid-latitudes by the 3-h $K p$ magnetic disturbance index, while the asymmetric and symmetric ring current indices, $A S Y-H$, $S Y M-H$, as well as Dst, are used as proxies of low-latitude geomagnetic activity. It has been shown (e.g., Stauning 2007, 2012; Stauning et al. 2008) that these parameters and indices, with a reasonable precision for space weather applications, could be derived from the two $P C$ indices and with degraded accuracy even from just one series, e.g., the $P C N$ indices.

Thus, with an average delay of $5 \mathrm{~min}$, the auroral electrojet $A E$ index is given (Stauning 2012) by

$$
A E=110 P C C+60(\mathrm{nT}) .
$$

The auroral electrojet activity comprises sudden enhancements related to the onset of substorms (auroral break-up). For a $P C$ index level below 2 there is hardly any substorm onset, for $P C$ increasing to levels between 2 and 5 , the substorm is 
delayed by $60-0 \mathrm{~min}$, while for $P C$ increasing to levels above 5 the substorm follows immediately (Janzhura et al. 2007).

The cross-polar cap potential could well be represented by an expression involving the $P C C$ index (Stauning 2012),

$$
\Phi_{\mathrm{PC}} \approx 20 P C C+15(\mathrm{kV}) .
$$

From the study by Chun et al. (1999), the total Joule heating power for the northern hemisphere $(J H N)$ was estimated and compared with the corresponding values of $P C N$. Their result for all data is reproduced in Eq. (9),

$$
J H N=4.03 P C N^{2}+27.3 P C N+7.7(\mathrm{GW}) .
$$

From Thule $P C N$ data and hemispheric auroral power data based on measurements from NOAA POES satellites in the period 1999-2002, and selecting only the northern passes, the Auroral Power $(A P N)$ index is given by (Stauning 2012):

$$
\begin{aligned}
& \text { Positive PCN: } A P N=13 \cdot P C N+10(\mathrm{GW}), \\
& \text { Negative } P C N: A P N=2.0 P C N+10(\mathrm{GW}) .
\end{aligned}
$$

Since different magnetospheric regions are linked to each other through magnetospheric currents, it is feasible to find relationships between magnetic indices and parameters that monitor solar wind effects at different latitudes. For global features this is better done using the $P C C$ index rather than individual $P C N$ or PCS indices.

With an average delay of $15 \mathrm{~min}$, the asymmetric 1-min ring current index, $A S Y-H$, usually based on low-latitude geomagnetic $\mathrm{H}$ component data, can be derived from the $P C C$ index through (Stauning et al. 2008; Stauning 2012):

$$
A S Y-H=12.1 P C C+11.5(\mathrm{nT}),
$$

with a standard deviation in $A S Y-H$, derived from Eq. (11), of $18 \mathrm{nT}$.

The 1-min symmetric ring current indices, $S Y M-H$, and the hourly $D s t$ index represent the energy stored in the ring current. These indices could be derived by integration of the $P C$ index considered a source function. In the analysis of Stauning (2007), the source function, $Q$, in Eq. (1) is expressed as a linear function of $P C C$,

$$
Q_{\mathrm{eq}}=4.6 P C C+1.2(\mathrm{nT} / \mathrm{h}) \text {. }
$$

When deriving Dst by integrating Eq. (1) and using the source function in Eq. (12), the fit to the real Dst values is as good as that obtained when using the merging electric field $E_{\mathrm{M}}$, Eq. (3), derived from satellite measurements and better than that obtained by taking into account the $E_{y}$ GSM component. Even more importantly, during the strongest storms in situ solar wind data may not be available or attain false values. Thus, using the polar cap indices (even just from one hemisphere, e.g., $P C N$ ) ensures a reliable back-up to providing reasonably accurate values of the instantaneous Dst (or SYM-H) indices. This provides a significant argument in support of using the $P C$ index for monitoring the magnetospheric activity, because it is a reliable proxy capable of characterising the solar wind energy that has entered into the magnetosphere.

These examples indicate the usefulness of polar cap indices to substantiate or even replace other parameters or indices used in the handling of space weather-dependent operational tasks, for instance the warning of major substorms and their possible GIC effects, or the calculation of thermospheric heating for prediction of satellite orbits. Details on the reliability and limits of applicability of the $P C$ indices are provided in the referenced sources, most comprehensively in the book chapter by Stauning (2012). The same book also holds an additional informative chapter on $P C$ indices written by Troshichev (2012).

\subsubsection{Response of field-aligned currents and the ionosphere to the solar wind}

Field-aligned currents, known also as Birkeland currents, are essential to the coupling between the solar wind-magnetosphere system and the ionosphere. Intensified FAC sheets, which emerge from the solar wind-magnetosphere interaction, are the primary cause of geomagnetic (sub)storms.

The generated small- and large-scale FACs and wave processes are responsible for the important Joule heating at ionosphere heights. In a pioneering study, Forget et al. (1991) theoretically examined the problem of ionosphere closure of small-scale FACs and found that the distribution of the Pedersen current that closes FACs depends on FAC scale. Their results imply that the Pedersen conductance is constant and equal to the classic integrated Pedersen conductance for FAC scales larger than $5 \mathrm{~km}$. On smaller scales, however, it shows a steep decrease down to the smallest scales. Hence, the closure of FACs through the Pedersen current and their Joule dissipation is redistributed in height according to their scale.

On the one hand, the FAC and wave activities are highly dependent on solar wind and IMF conditions. On the other hand, the rate of Joule heating is an intrinsic property of the ionosphere-thermosphere system and depends on the ionosphere state and its conductivity, the ion-neutral collision frequency, the neutral wind conditions, etc. In this paper, an example of FAC dynamics and basic ionospheric parameter variations in height illustrates this competitive interaction.

Both EISCAT and CHAMP satellite data were used to compare FAC dynamics and ionosphere parameter variations under quiet conditions. EISCAT measurements of the ionosphere (UHF radar, Tromsø, Norway) were obtained between 30 June and 2 July 2008, and FAC measurements conducted on board the CHAMP satellite are from orbits crossing approximately over Tromsø during that time. The EISCAT measurements were conducted in three morning and two evening sessions of $4 \mathrm{~h}$ each. Data were processed to derive average magnitudes of the plasma concentration, $N_{\mathrm{e}}$, electron and ion-temperatures, $T_{\mathrm{e}}$ and $T_{\mathrm{i}}$, and the ion-drift velocity, $V_{\text {drift, }}$, for each session. Ionosphere parameters shown in Figures 6 and 7 (Teodosiev et al. 2011) refer to the 1 July 2008 EISCAT experiment (Fig. 6 for the morning session and Fig. 7 for the evening session). The magnitude of the $B_{x}$ component of the IMF varies between -5 and $+4 \mathrm{nT}$, while $B_{y}$ and $B_{z}$ variations are between -2 and $+3 \mathrm{nT}$ (for $B_{y}$ ) and between -1 and $+2 \mathrm{nT}$ (for $B_{z}$ ). For the period of interest, the $K p$ index was $1-(09-12$ UT) and $0+(21-24$ UT). The possible large-scale FAC distribution was calculated based on the measured solar wind and IMF parameters. The FAC model used was calculated from the Nenovski's (2008) model. Two sets of solar wind and IMF parameters were chosen during the evening session at 23 UT. Approximately the same FAC distribution is derived from the Weimer's (2005) model.

The EISCAT experiment reveals a maximum of the ion (electron) temperature, $T_{\mathrm{i}}\left(T_{\mathrm{e}}\right)$, at heights of $200-250 \mathrm{~km}$ in both the noon and midnight sectors. Such atypical increases (of up to $2000 \mathrm{~K}$ ) have been observed in the night sector under disturbed conditions (see Schunk \& Zhu 2008); a similar effect, 

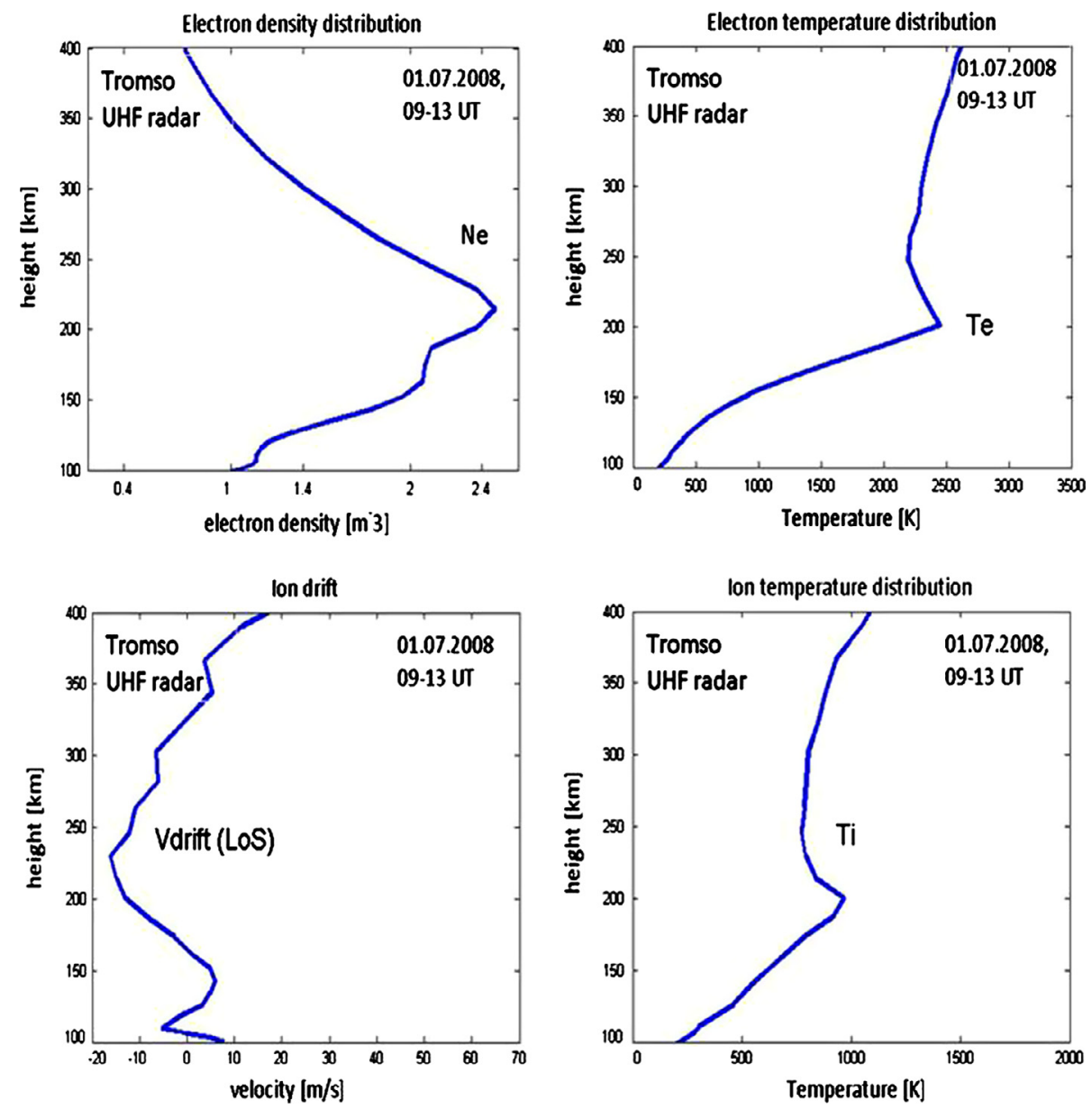

Fig. 6. Ionospheric parameters distribution as a function of height for the morning session on day 182 (1 July 2008) for each of the four quantities. Data in the height interval of $100-400 \mathrm{~km}$ only are shown.

however, is observed under quiet conditions. The electron density maximum is at approximately the same heights. The ion drift is negative $\left(\sim-10 \mathrm{~m} \mathrm{~s}^{-1}\right)$ at heights between 120 and $300 \mathrm{~km}$ both in day and night conditions. However, this drift differs at heights $>300 \mathrm{~km}$ : positive (away from the radar) during the morning session and negative (towards the radar) in the evening. Note that the negative ion drift observed by EISCAT does not confirm the large-scale FAC direction from the FAC models (Teodosiev et al. 2011).

One possible explanation of the observed ion-drift direction is the neutral wind influence exerted on the $\mathrm{O}^{+}$ions. Another explanation is possible: this drift can be interpreted as downward ambipolar diffusion.

The orbits of CHAMP satellite passing over Tromsø are similarly examined. The FAC intensity (averaged over intervals of $20 \mathrm{~s}$ ) at Tromsø appears to be very low: its magnitude is approximately $0.1 \mu \mathrm{A} \mathrm{m}^{-2}$. This suggests that the electric field strength in the ionosphere that closes FAC (at Tromsø) amounts to $5 \mathrm{mV} \mathrm{m}^{-1}$ or less; the classic Pedersen conductivity using EISCAT data is approximately $2 \times 10^{-4} \mathrm{~S} \mathrm{~m}^{-1}$.

Simple estimations show that only FAC densities higher than $10^{-4} \mu \mathrm{A} \mathrm{m}^{-2}$ and electric fields, $E$, of $50-100 \mathrm{mV} \mathrm{m}^{-1}$ could account for ion-temperature increases of order $100 \mathrm{~K}$ as observed by EISCAT. Forget's et al. (1991) model results and estimations suggest that the main contribution to Joule dissipation at high latitudes (which is thought responsible for initiating ion-temperature increases at heights of 200-250 km) should come from intense FAC structures on smaller scales.

Another finding is that the summer-winter FAC differences (revealed by CHAMP) will significantly influence the global patterns of Joule heating (Zhang et al. 2005). Indeed, the ion velocity shows a maximum in winter and a minimum in summer. This corresponds to a maximum (in summer) and a minimum (in fall) of the squared ion velocity that has been found experimentally. It has been shown that during solar minimum conditions, the $\mathrm{F}$ region contributes less than $20 \%$ to the total height-integrated Pedersen conductivity. In contrast, during solar maximum conditions, the contribution to the Pedersen conductance from solar produced F-region ionisation can be 60\% (De la Beaujardiere et al. 1991).

EISCAT experiments and CHAMP data demonstrate that the temperature increase, $\Delta T$, maximises mainly at latitudes between either FAC regions 1 or 2, where the electric field is intensified and the Pedersen conductance is high, and at heights close to the heights of the foF 2 frequency, $h^{\prime} \mathrm{F} 2 \max$.

The close connection between $\Delta T$ and the height of the foF 2 frequency might be further validated. If this process results in formation of a neutral density bulge that propagates outside of the FAC region, an increase/decrease of the F2 ionisation at the same heights will be a consequence. Thus, a foF 2 frequency 

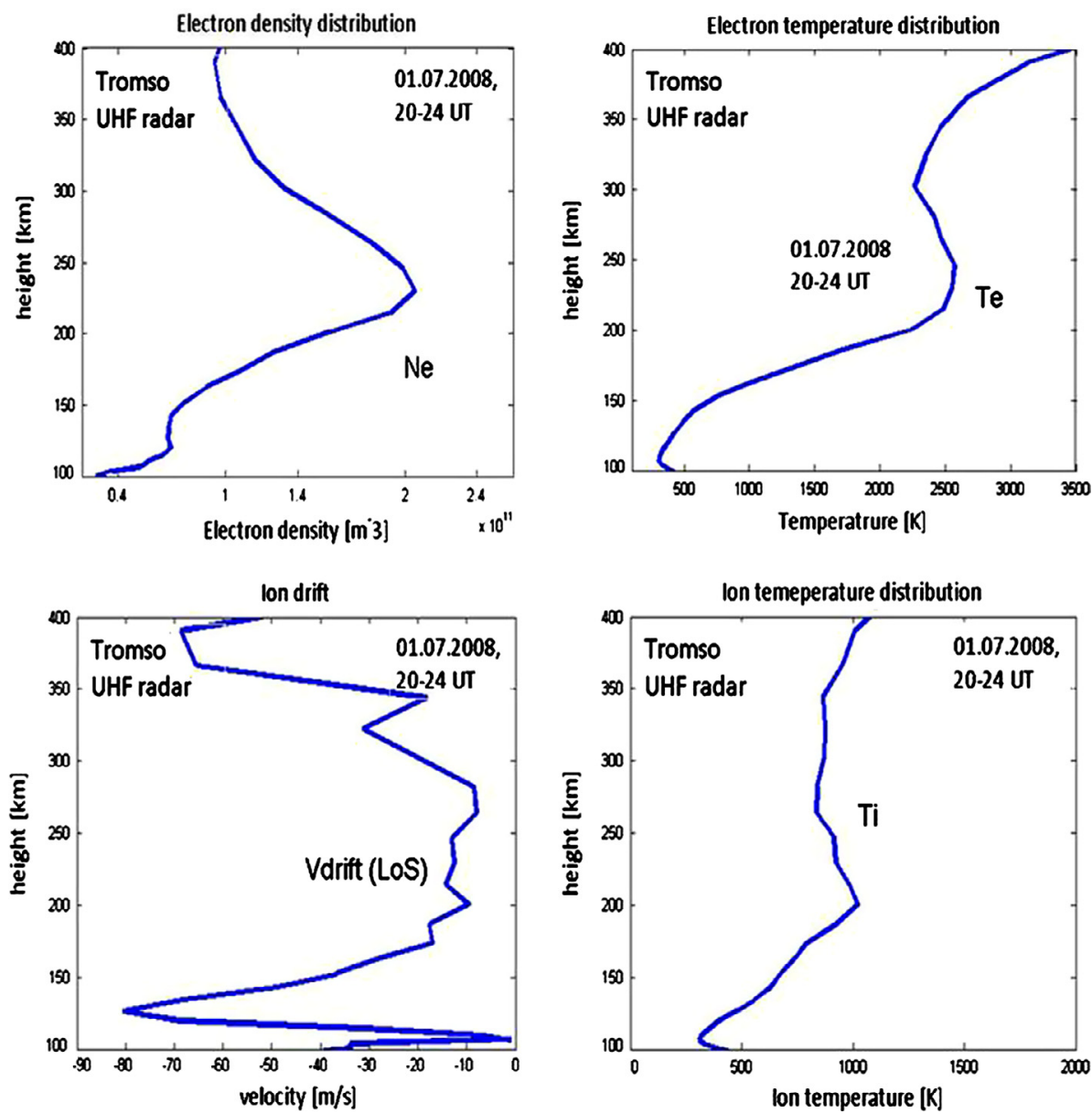

Fig. 7. Ionospheric parameters distribution as a function of height for the evening session on day 182 (1 July 2008) for each of the four quantities. Data in the height interval of $100-400 \mathrm{~km}$ only are shown.

increase/decrease is seen after every FAC intensity change, which can be modelled based on the solar wind and IMF parameter variations.

The following conclusions can be drawn. (i) Even under quiet geomagnetic activity, the ionosphere response is highly dependent on processes on much smaller spatial and temporal scales, e.g., scales smaller or shorter than a few kilometres or seconds. (ii) Thorough understanding of the physics of Joule heating processes at high latitudes under various geomagnetic conditions requires complete monitoring of small-scale FACstructure dynamics and ionospheric parameter variations over a wide range of solar wind and IMF parameters.

\section{Summary and conclusions}

Since the end of the 20th century, when the space weather discipline was born, much effort has been dedicated to obtain the best predictions. Lots of technological applications (radio communications, navigation systems, power grids, etc.) depend on the state of the magnetosphere, the ionosphere or the ground, which may be strongly affected by the arrival of electromagnetic radiation, energetic particles and interplanetary structures during space weather events. Many models have been developed, some based on physical laws, others on empirical or semi-empirical results, and each intended to reproduce the range of phenomena involved in only part of the complete, extremely complex reality.
In the framework of the COST Action ES0803 "Developing space weather products and services in Europe", scientists from several European countries have addressed practical problems in space weather research. This article reviews a collection of achievements of some COST Action ES0803 participants, which constitutes a useful contribution to space weather community because either improvements on modelling schemes or physical insights can be highlighted. The influence that improvements in the field would have for various practical purposes is unquestionable and therefore a forecaster should take into account the information about the topics addressed in this paper.

The main conclusions on the progress achieved are summarised below. It is well known that solar variability has had a noticeable influence on the Earth's climate on timescales of decades. However, the solar luminosity, variability of the solar ultraviolet radiation and other cycle-dependent variations are not yet well understood, mainly because direct measurements of the relevant quantities have only recently become possible. As regards the long-term variability of interplanetary and geomagnetic magnitudes, MC and GC signals exhibit their solar origin.

In relation to forecasting whether a solar X-ray flare will be followed by a severe geomagnetic disturbance, the highest value of the contingency coefficient $C$ was achieved when the input parameters for the artificial neural network were the heliographic longitude and latitude, Type II and/or IV RSPs, 
XRA class and $\Delta \log (\Phi)$. This shows the positive impact on the prediction scheme of additional information on the enhancement of the $\geq 10 \mathrm{MeV}$ high-energy proton flux in the 10-h time window following the 12-h after the XRA occurrence.

The solar wind-magnetospheric response coupling has highlighted the enhanced geoeffectiveness resulting from interactions among different outcomes from the solar activity, whose signatures in the interplanetary medium are large solar wind density enhancements and large changes to the southward direction of the $B_{z}$ component of the IMF. These interplanetary signatures are responsible for sharp decreases in Dst.

The need to provide a new injection function from the solar wind to the magnetosphere was also indicated from a physical point of view to explain the temporal evolution of the Dst index when the main phase of the storm develops on short timescales. As extreme events fit into that category, this research line should be a priority for researchers.

Likewise, nonlinear behaviour of magnetospheric recovery has been proven. As a result, a hyperbolic function is provided, which properly reproduces the entire recovery phase as traced by the $D s t$ index. Although the physical implications for both terms in the energy-balance equation of the ring current (source and loss terms) are still being studied, a new generation of models will be developed to describe the temporal evolution of the Dst index.

A combined $P C$ index $(P C C)$ has been proposed to avoid the different responses of the two polar caps to solar wind forcing. This index provides a useful characterisation of the state of the polar ionosphere and can be considered a reliable proxy for the solar wind merging electric field, even during strong and northward IMF conditions, since it is more representative of the global disturbance level than the individual $P C N$ or $P C S$ indices. Moreover, parameters and indices that monitor the influence of the solar wind energy input in the high-latitude magnetosphere could be derived from the $P C C$ index and can, therefore, be used for space weather applications. Warnings of substorms and their possible effects, the calculation of thermospheric heating for prediction of satellite orbits, are only a few of the potential resources. Even under quiet geomagnetic activity, the ionospheric response is highly dependent on processes on much smaller spatial and temporal scales, for example, on scales smaller than a few kilometres and/or less than a few seconds. Therefore, a thorough understanding of the physics of Joule heating processes at high latitudes under various geomagnetic conditions requires complete monitoring of small-scale FAC-structure dynamics and ionospheric parameter variations over a wide range of solar wind and IMF parameters.

Finally, we conclude that a European space weather research community has been established. Although that community has progressed substantially, more effort is required to gain a better and deeper understanding of the underlying physical processes. This progress will provide improved models and, as a consequence, a greater prediction capability for Europe. The results discussed in this paper can be considered as proof of the success of COST Action ES0803 and the need of other collaborative activities to further advance the space weather discipline.

Acknowledgements. We gratefully acknowledge the financial support provided by the EU COST office to COST Action ES0803, which provided the support for an interdisciplinary network of European scientists dealing with different issues related to geospace. ES, YC and CC acknowledge support from the Spanish Projects
PN-AYA2009-08662 from the Ministerio de Ciencia e Innovación and PPII10-0183-7802 from the Junta de Comunidades de Castilla-La Mancha. PH, DT and FV acknowledge support from the Ministry of Education, Youth and Sports of the Czech Republic under the Project OC09070 and by VEGA Grants 2/0015/11 and 2/0022/11 of the Scientific Grant Agency of the Ministry of Education of the Slovak Republic and the Slovak Academy of Sciences. All authors are grateful for use of the database used for the preparation of this review paper.

\section{References}

Aguado, J., C. Cid, E. Saiz, and Y. Cerrato, Hyperbolic decay of the Dst index during the recovery phase of intense geomagnetic storms, J. Geophys. Res., 115, A07220,

DOI: 10.1029/2009JA014658, 2010.

Amory-Mazaudier, C., Sun Earth's System: the transient variations of the Earth's magnetic field. Edited by A. Hady, and M.I. Wanas, First Middle East-Africa, Regional IAU Meeting, Proceedings MEARIM, 1, 236-270, DOI: 10.1017/97740330200173, 2008.

Andreasen, G., Reconstruction of past solar wind variations: inversion of the geomagnetic response at Godhavn, J. Geophys. Res., 102, A4, 7025-7036, 1997.

Baumjohann, W., and R.A. Treumann, Basic Space Plasma Physics, Covent Garden, London: Imperial College Press, 103-128, ISBN: 1-86094-079-X, 1997.

Boberg, F., P. Wintoft, and H. Lundstedt, Real time Kp predictions from solar wind data using neural networks, Phys. Chem. Earth, 25, 275-280, 2000.

Bochníček, J., and P. Hejda, Areas of minimum intensity of soft Xrays as sources of solar wind high-speed streams, J. Atmos. Sol. Terr. Phys., 64, 511-515, 2002.

Bochníček, J., P. Hejda, and F. Valach, Solar energetic events in the years 1996-2004. The analysis of their geoeffectiveness, Stud. Geophys. Geod., 51 (3), 439-447, DOI: 10.1007/s11200-007-0025-4, 2007.

Bogdanova, Y.V., et al., Formation of the low-latitude boundary layer and cusp under the northward IMF: simultaneous observations by Cluster and Double Star, J. Geophys. Res., 113, A07S07, 1-33, DOI: 10.1029/2007JA012762, 2008.

Borovsky, J.E., and M.H. Denton, Differences between CME-driven storms and CIR-driven storms, J. Geophys. Res., 111, A07S08, DOI: 10.1029/2005JA011447, 2006.

Burton, R.K., R.L. McPherron, and C.T. Russell, An empirical relationship between interplanetary conditions and Dst, J. Geophys. Res., 80 (31), 4204-4214, DOI: 10.1029/JA080i031p04204, 1975.

Caballero-Lopez, R., A.H. Moraal, K.G. McCracken, and F.B. McDonald, The heliospheric magnetic field from 850 to $2000 \mathrm{AD}$ inferred from ${ }^{10} \mathrm{Be}$ records, J. Geophys. Res., 109, A12102, DOI: 10.1029/2004JA010633, 2004.

Campbell, W.H., Introduction to geomagnetic fields, 2nd Edn., Cambridge, UK: Cambridge University Press, 337 pp., 2003.

Cane, H.V., R.G. Stone, J. Fainberg, J.L. Steinberg, and S. Hoang, Type II solar radio events observed in the interplanetary medium I: general characteristics, Sol. Phys., 78, 187-198, 1982.

Cerrato, Y., E. Saiz, C. Cid, W. D. Gonzalez, and J. Palacios, Solar and interplanetary triggers of the largest Dst variations of the solar cycle 23, J. Atmos. Sol. Terr. Phys., 80, 111-123, DOI: 10.1016/j.jastp.2011.09.001, 2011.

Chisham, G., M.P. Freeman, T. Sotirelis, R.A. Greenwald, M. Lester, and J.-P. Villain, A statistical comparison of SuperDARN spectral width boundaries and DMSP particle precipitation boundaries in the morning sector ionosphere, Ann. Geophys., 23, 733-743, DOI: 10.5194/angeo-23-733-2005, 2005.

Chun, F.K., D.J. Knipp, M. G. McHarg, G. Lu, B.A. Emery, S. Vennerstrøm, and O.A. Troshichev, Polar cap index as a proxy for hemispheric Joule heating, Geophys. Res. Lett., 26, 1101-1104, 1999. 
Cid, C., H. Cremades, A. Aran, C. Mandrini, B. Sanahuja, B. Schmieder, M. Menvielle, L. Rodriguez, E. Saiz, Y. Cerrato, et al., Can a halo CME from the limb be geoeffective, J. Geophys. Res., 117, 25, A11102, DOI: 10.1029/2012JA017536, 2012.

Cid, C., J. Palacios, E. Saiz, Y. Cerrato, J. Aguado, and A. Guerrero, Modeling the recovery phase of the extreme geomagnetic storms, $J$. Geophys. Res., 118, 1-8, DOI: 10.1002/jgra.50409, 2013 (under review).

Cliver, E.W., V. Boriakoff, and K.H. Bounar, The 22-year cycle of geomagnetic activity, J. Geophys. Res., 101, 27091-27109, 1996.

Cliver, E.W., V. Boriakoff, and J. Feynman, Solar variability and climate change: geomagnetic $a a$ index and global surface temperature, Geophys. Res. Lett., 25, 1035-1038, 1998.

Clilverd, M.A., E. Clarke, T. Ulich, J. Linthe, and H. Rishbeth, Reconstructing the long-term $a a$ index, J. Geophys. Res., 110, A07205, DOI: 10.1029/2004JA010762, 2005.

Crooker, N.U., J.T. Gosling, V. Bothmer, R.J. Forsyth, P.R. Gazis, A. Hewish, T.S. Horbury, D.S. Intriligator, J.R. Jokipii, J. Kóta, et al., Turbulence, discontinuities, and energetic particles, Space Sci. Rev., 89, 179-220, DOI: 10.1023/A:1005253526438, 1999.

Daglis, I.A., R.M. Thorne, W. Baumjohann, and S. Orsini, The terrestrial ring current: origin, formation, and decay, Rev. Geophys., 37 (4), 407-438, DOI: 10.1029/1999RG900009, 1999.

Dasso, S., D. Gómez, and C.H. Mandrini, Ring current decay rates of magnetic storms: a statistical study from 1957 to $1998, J$. Geophys. Res., 107 (A5), 1059, DOI: 10.1029/2000JA000430, 2002.

Dasso, S., C.H. Mandrini, B. Schmieder, H. Cremades, C. Cid, Y. Cerrato, E. Saiz, P. De'moulin, A.N. Zhukov, L. Rodriguez, A. Aran, M. Menvielle, and S. Poedts, Linking two consecutive nonmerging magnetic clouds with their solar sources, J. Geophys. Res., 114, A02109, DOI: 10.1029/2008JA013102, 2008.

De Keyser, J., M.W. Dunlop, C.J. Owen, B.U.Ö. Sonnerup, S.E. Haaland, A. Vaivads, G. Paschmann, R. Lundin, and L. Rezeau, Magnetopause and Boundary Layer, Space Sci. Rev., 118, 231320, DOI: 10.1007/s11214-005-3834-1, 2005.

De la Beaujardiere, O., R. Johnson, and V.B. Wickwar, , Groundbased measurements of Joule heating rates. Edited by C.-I. Meng, M.J. Rycroft, and L.A. Frank, Auroral Physics, Cambridge, England: Cambridge University Press, 436-448, ISBN-13: 9780521157414, 1991.

Demetrescu, C., and V. Dobrica, Signature of Hale and Gleissberg solar cycles in the geomagnetic activity, J. Geophys. Res., 113, A02103, DOI: 10.1029/2007JA012570, 2008.

Demetrescu, C., V. Dobrica, and G. Maris, On the long-term variability of the heliosphere-magnetosphere environment, Adv. Space Res., 46, 1299-1312, DOI: 10.1016/j.asr.2010.06.032, 2010.

Dessler, A.J., and E.N. Parker, Hydromagnetic Theory of Geomagnetic Storms, J. Geophys. Res., 64, 2239-2252, 1959.

$\mathrm{Du}$, Z.L., The correlation between solar and geomagnetic activity Part 2: long-term trends, Ann. Geophys., 29, 1341-1348, DOI: 10.5194/angeo-29-1341-2011, 2011.

Du, A.M., B.T. Tsurutani, and W. Sun, Anomalous geomagnetic storm of 21-22 January 2005: a storm main phase during northward IMFs, J. Geophys. Res., 113, A10214, DOI: 10.1029/2008JA013284, 2008.

Dungey, J.W., Interplanetary magnetic field and the auroral zones, Phys. Rev. Lett., 6, 47-48, 1961.

Dunlop, M.W., Q.-H. Zhang, C.-J. Xiao, J.-S. He, Z. Pu, R.C. Fear, C. Shen, and C.P. Escoubet, Reconnection at high latitudes: antiparallel merging, Phys. Rev. Lett., 102, 075005, 2009.

Ebihara, Y., M. Ejiri, and H. Miyaoka, Coulomb lifetime of the ring current ions with time varying plasmasphere, Earth Planets Space, 50 (4), 371-382, 1998.

Echer, E., W.D. Gonzalez, A.L.C. Gonzalez, A. Prestes, L.E.A. Vieira, A. Dal Lago, F.L. Guarnieri, and N.J. Schuch, Long-term correlation between solar and geomagnetic activity, J. Atmos. Sol. Terr. Phys., 66, 1019-1025, 2004.
Eddy, J.A., The case of the missing sunspots, Sci. Am., 236 (5), 8092, DOI: 10.1038/scientificamerican0577-80, 1977.

Fejer, B., and L. Scherliess, Empirical models of storm time equatorial zonal electric fields, J. Geophys. Res., 102 (A11), 24047-24056, DOI: 10.1029/97JA02164, 1997.

Feldstein, Y.I., and L.A. Dremukhina, On the two-phase decay of the Dst variation, J. Geophys. Res., 27 (17), 2813-2816, 2000.

Feynman, J., and N.U. Crooker, The solar wind at the turn of the century, Nature, 275, 626, 1978.

Feynman, J., and A. Ruzmaikin, The Sun's strange behavior: Maunder minimum or Gleissberg cycle?, Sol. Phys., 272 (2), 351-363, DOI: 10.1007/s11207-011-9828-0, 2011.

Finch, I., and M. Lockwood, Solar wind-magnetosphere coupling functions on timescales of 1 day to 1 year, Ann. Geophys., 25, 495-506, DOI: 10.5194/angeo-25-495-2007, 2007.

Forget, B., J.-C. Cerisier, A. Berthelier, and J.-J. Bertheleier, Ionospheric closure of small-scale Birkeland currents, J. Geophys Res., 96 (A2), 1843-1847, DOI: 10.1029/90JA02376, 1991.

Foster, J.C., and F.J. Rich, Prompt mid-latitude electric fields effects during severe geomagnetic storms, J. Geophys. Res., 103 (11), 26367-26372, DOI: 10.1029/97JA03057, 1998.

Fuselier, S.A., J. Berchem, K.J. Trattner, and R. Friedel, Tracing ions in the cusp and low-latitude boundary layer using multispacecraft observations and a global MHD simulation, J. Geophys. Res., 107 (A9), 1226, DOI: 10.1029/2001JA000130, 2002.

Gleisner, H., and H. Lundstedt, A neural network-based local model for prediction of geomagnetic disturbances, J. Geophys. Res., 106, 8425-8434, 2001

Gleisner, H., and H. Lundstedt, Auroral electrojet predictions with dynamic neural network, J. Geophys. Res., 106, 24541-24550, 2001.

Gleisner, H., and J. Watermann, Solar energetic particle flux enhancement as an indicator of halo coronal mass ejection geoeffectivness, Space Weather - Inter. J. Res. Appl., 4, S06006, DOI: $10.1029 / 2006$ SW000220, 2006a.

Gleisner, H., and J. Watermann, Concepts of medium-range (1-3 days) geomagnetic forecasting. Space weather prediction: applications and validation, Adv. Space Res., 37, 1116-1123, $2006 \mathrm{~b}$.

Gopalswamy, N., Coronal Mass Ejections and Solar Radio Emissions. Edited by H. Rucker, W. Kurth, P. Louarn, and G. Fischer, Proceedings of the 7th International Workshop on Planetary, Solar and Heliospheric Radio Emissions (PRE VII), 325-342, ISBN: 978-3-7001-7125-6, 2011.

Hamilton, D.C., G. Gloeckler, F.M. Ipavich, W. Stüdemann, B. Wilken, and G. Kremser, Ring current development during the great geomagnetic storm of February 1986, J. Geophys. Res., 93 (12), 14343-14355, DOI: 10.1029/JA093iA12p14343, 1988.

Hasegawa, H., et al., Rolled-up Kelvin-Helmholtz vortices and associated solar wind entry at Earth's magnetopause, Nature, 430, 755-758, 2004

Hosokawa, K., E.E. Woodfield, M. Lester, S.E. Milan, N. Sato, A.S. Yukimatu, and T. Iyemori, Interhemispheric comparison of spectral width boundary as observed by SuperDARN radars, Ann. Geophys., 21, 1553-1565, 2003.

Hughes, W.J., and M.K. Hudson, Towards an integrated model of the space weather system, J. Atmos. Sol. Terr. Phys., 66, 1241-1242, DOI: 10.1016/j.jastp.2004.06.001, 2004.

Imber, S.M., S.E. Milan, and M. Lester, The Heppner-Maynard Boundary measured by SuperDARN as a proxy for the latitude of the auroral oval, J. Geophys. Res. Space Phys., 118, 685-697, DOI: 10.1029/2012JA018222, 2013.

Janzhura, A., O.A. Troshichev, and P. Stauning, Unified PC indices: relation to isolated magnetic substorms, J. Geophys. Res., 112, A09207, DOI: 10.1029/2006JA012132, 2007.

Johnson, J., and C. Cheng, Kinetic Alfven waves and plasma transport at the magnetopause, Geophys. Res. Lett., 242, 1423, DOI: 10.1029/97GL01333, 1997.

Jordanova, V.K., L.M. Kistler, J.U. Kozyra, G.V. Kharanov, and A.F. Nagy, Collisional losses of ring current ions, J. Geophys. Res., 101, 111-126, 1996. 
Jordanova, V.K., A. Boonsiriseth, R.M. Thorne, and Y. Dotan, Ring current asymmetry from global simulations using a high-resolution electric field model, J. Geophys. Res., 108 (12), 1443-1443, DOI: 10.1029/2003JA009993, 2003.

Kan, J.R., and L.C. Lee, Energy coupling function and solar windmagnetosphere dynamo, Geophys. Res. Lett., 6 (7), 577-580, DOI: 10.1029/GL006i007p00577, 1979.

Kelley, M.C., The Earth's ionosphere: plasma physics and electrodynamics, Intern. Geophys. Ser., San Diego: Academic Press, 1989.

Kelley, M.C., and J. Retterer, First successful prediction of a convective equatorial ionospheric storm using solar wind parameters, Space Weather, 6, S08003, 4 pp. DOI: 10.1029/2007SW000381, 2008.

Kim, R.S., K.S. Cho, Y.J. Moon, Y.H. Kim, Y. Yi, M. Dryer, S.C. Bong, and Y.D. Park, Forecast evaluation of the coronal mass ejection (CME) geoeffectiveness using halo CMEs from 1997 to 2003, J. Geophys. Res., 110, A11104, DOI: 10.1029/2005JA011218, 2005.

Kosugi, T., and K. Shibata, Solar coronal dynamics and flares as a cause of interplanetary disturbances, in Magnetic Storms. Edited by B.T. Tsurutani, W.D. Gonzalez, Y. Kamide, and J.K. Arballo, Geophysical Monograph, 98, Washington D.C: AGU Press, 21-34, 1997.

Kozyra, J.U., and M.W. Liemohn, Ring current energy input and decay, Space Sci. Rev., 109, 105-131, DOI: 10.1023/B: SPAC.0000007516.10433.ad, 2003.

Kozyra, J.U., V.K. Jordanova, R.B. Home, and R.M. Thorne, Magnetic Storms. Edited by B.T. Tsurutani, et al., Geophys. Monogr. Ser., Vol. 98, Washington DC: AGU,

DOI: $10.1029 / \mathrm{GM} 098$, ISSN: 0065-8448; ISBN: 0-87590-0801, 187-202, 1997.

Kozyra, J.U., M.-C. Fok, E.R. Sanchez, D.S. Evans, D.C. Hamilton, and A.F. Nagy, The role of precipitation losses in producing the rapid early recovery phase of the great magnetic storm of February 1986, J. Geophys. Res., 103 (A4), 6801-6814, DOI: 10.1029/97JA03330, 1998.

Kozyra, J.U., M.W. Liemohn, C.R. Clauer, A.J. Ridley, M.F. Thomsen, J.E. Borovsky, J.L. Roeder, V.K. Jordanova, and W.D. Gonzalez, Multistep Dst development and ring current composition changes during the 4-6 June 1991 magnetic storm, $J$. Geophys. Res., 107 (8), 1224, DOI: 10.1029/2001JA000023, 2002.

Krivova, N.A., L. Balmaceda, and S.K. Solanki, Reconstruction of solar total irradiance since 1700 from the surface magnetic flux, $A \& A, \quad 467, \quad 335-346, \quad$ DOI: 10.1051/0004-6361:20066725, 2007.

Kuklin, G.V., Cyclical and secular variations of solar activity. Edited by V. Bumba, and J. Kleczek, Basic mechanisms of solar activity, IAU Symposium No. 71, Dordrecht-Holland/Boston, USA: D. Reidel Publishing Company, 147-190, ISBN-10: 9027706808, ISBN-13: 9789027706805, 1976.

Lavraud, B., M.F. Thomsen, B. Lefebvre, S.J. Schwartz, K. Seki, T.D. Phan, Y.L. Wang, A. Fazakerley, H. Rème, and A. Balogh, Evidence for newly closed magnetosheath field lines at the dayside magnetopause under northward IMF, J. Geophys. Res., 111, A05211, DOI: 10.1029/2005JA011266, 2006.

Le Mouël, J.-L., V. Kossobokov, and V. Courtillot, On long-term variations of simple geomagnetic indices and slow changes in magnetospheric currents: the emergence of anthropogenic global warming after 1990?, Earth Planet. Sci. Lett., 232, 273-286, 2005 .

Lean, J., Evolution of the Sun's spectral irradiance since the Maunder Minimum, Geophys. Res. Lett., 22, 2425-2428, 2000.

Lean, J., J. Beer, and R. Bradley, Reconstruction of solar irradiance since 1610: implications for climate change, Geophys. Res. Lett., 22, 3195-3198, 1995.

Lester, M., S.E. Millan, G. Provan, and J.A. Wild, Review of the ionospheric effects of solar wind magnetosphere coupling in the context of the expanding contracting polar cap boundary model, Space Sci. Rev., 124, 117-130, 2006.

Liemohn, M.W., and J.U. Kozyra, Testing the hypothesis that charge exchange can cause a two-phase decay. Edited by M. Burch, J.L. Burch, M. Schulz, and H. Spence, Inner Magnetosphere Interactions: New Perspectives from imaging, 159, Washington, D.C: AGU, Geophys. Monogr. Ser., ISBN: 978-0-87590-424-5, 67-178, 2005

Liemohn, M.W., J.U. Kozyra, C.R. Clauer, and A.J. Ridley, Computational analysis of the near-Earth magnetospheric current system during two-phase decay storms, J. Geophys. Res., 106 (A12), 29531-29542, DOI: 10.1029/2001JA000045, 2001.

Lockwood, M., R. Stamper, and M.N. Wild, A doubling of the Sun's coronal magnetic field during the past 100 years, Nature, 399 , 437-439, 1999.

Lockwood, M., A.P. Rouillard, and I.D. Finch, The rise and fall of open solar flux during the current grand solar maximum, Astrophys. J., 700, 937-944, DOI: 10.1088/004-637x/700/2/937, 2009.

Lopez, R.E., M. Wiltberger, S. Hernandez, and J.G. Lyon, Solar wind density control of energy transfer to the magnetosphere, Geophys. Res. Lett., 31, L08804, DOI: 10.1029/2003GL018780, 2004.

Lundstedt, H., P. Wintoft, H. Gleisner, F. Boberg, T. Hasanov, and I. Kronfeldt, Forecasting space weather and effects using knowledge-based neurocomputing. In: Proceedings of Space Weather Workshop: Looping Towards a European Space Weather Programme, Noordwijk, The Netherlands: ESA Publishing Division, 179-184, WPP-194, 2002a.

Lundstedt, H., H. Gleisner, and P. Wintoft, Operational forecasts of geomagnetic Dst index, Geophys. Res. Lett., 29, 2181-2181, DOI: 10.1029/2002GL016151, 2002b.

Marcucci, M.F., I. Coco, D. Ambrosino, E. Amata, S.E. Milan, M.B. Bavassano Cattaneo, and A. Retinò, Extended SuperDARN and IMAGE observations for northward IMF: evidence for dual lobe reconnection, J. Geophys. Res., 113, 1-12, A02204, DOI: 10.1029/2007JA012466, 2008.

Masters, A., D.G. Mitchell, A.J. Coates, and M.K. Dougherty, Saturn's low-latitude boundary layer: 1. Properties and variability, J. Geophys. Res., 116, 1-13, A06210, DOI: 10.1029/2010JA016421, 2011.

McCracken, K.G., Heliomagnetic field near Earth, 1428-2005, J. Geophys. Res., 112, A09106, DOI: 10.1029/2006JA012119, 2007.

Menvielle, M., Geomagnetic indices in Geomagnetic Observations and Models. Edited by M. Mandea, and M. Korte, IAGA Special Sopron Book Series, Vol. 5, Springer Science+Business Media, 183-228, ISBN: 978-90-481-9857-3, DOI: 10.1007/978-90-481-9858-0 8, 2011.

Monreal MacMahon, R., and C. Llop, Ring current decay time model during geomagnetic storms: a simple analytical approach, Ann. Geophys., 26, 2543-2550, 2008

Mursula, K., I.G. Usoskin, and G.A. Kovaltsov, Persistent 22-year cycle in sunspot activity: evidence for a relic solar magnetic field, Sol. Phys., 198, 51-56, 2001.

Mursula, K., D. Martini, and A. Karinen, Did open solar magnetic field increase during the last 100 years? A reanalysis of geomagnetic activity, Sol. Phys., 224, 85-94, 2004.

Nelson, G.J., and D.B. Melrose, Type II bursts. Edited by D.J. MacLean, and N.R. Labrum, Solar Radiophysics: Studies of Emission from the Sun at Metre Wavelengths (A87-13851 03-92), New York: Cambridge Univ. Press, 333-359, ISBN: 978-0521254090, 1985.

Nenovski, P., Comparison of simulated and observed large-scale field-aligned current structures, Ann. Geophys., 26 (2), 281-293, 2008.

O'Brien, T.P., and R.L. McPherron, An empirical phase space analysis of ring current dynamics: solar wind control of injection and decay, J. Geophys. Res., 105 (4), 7707-7719, DOI: 10.1029/1998JA000437, 2000 
Oksavik, K., F. Søraas, J. Moen, and W.J. Burke, Optical and particle signatures of magnetospheric boundary layers near magnetic noon: satellite and ground-based observations, J. Geophys. Res., 105 (A12), 27555-27568, DOI: 101029/1999JA000237, 2000.

Phan, T.-D., M. Oieroset, and M. Fujimoto, Reconnection at the dayside low-latitude magnetopause and its nonrole in low-latitude boundary layer formation during northward interplanetary magnetic field, Geophys. Res. Lett., 32, 1-2, DOI: 10.1029/2005GL023355, 2005.

Pick, M., T.G. Forbes, G. Mann, H.V. Cane, J. Chen, A. Ciaravella, H. Cremades, R.A. Howard, H.S. Hudson, A. Klassen, et al., Multi-Wavelength Observations of CMEs and Associated Phenomena, Report of Working Group F, Space Sci. Rev., 123, 341, 2006.

Prölss, G.W., Common origin of positive ionospheric storms at middle latitudes and the geomagnetic activity effect at low latitudes, J. Geophys. Res., 98, 5981-5991, DOI: 10.1029/92JA02777, 1993.

Rangarajan, G.K., Indices of geomagnetic activity. Edited by J.A. Jacobs, Geomagnetism, Vol. 3, London: Academic Press, 385-460, 1989.

Reiner, M.J., M.L. Kaiser, J. Fainberg, J.-L. Bougeret, and R.G. Stone, On the origin of radio emissions associated with the January 6-11, 1997, CME, Geophys. Res. Lett., 25 (14), $2493-$ 2496, DOI: 10.1029/98GL00138, 1998.

Richardson, I.G., and H.V. Cane, Solar wind drivers of geomagnetic storms during more than four solar cycles, J. Space Weather Space Clim., 2, A01, DOI: 10.1051/swsc/2012001, 2012a.

Richardson, I.G., and H.V. Cane, Near-earth solar wind flows and related geomagnetic activity during more than four solar cycles (1963-2011), J. Space Weather Space Clim., 2, A02, DOI: $10.1051 / \mathrm{swsc} / 2012003,2012 \mathrm{~b}$.

Richardson, I.G., H.V. Cane, and E.W. Cliver, Sources of geomagnetic activity during nearly three solar cycles (1972-2000), J. Geophys. Res., 107, A8, DOI: 10.1029/2001JA00054, 2002.

Robbrecht, E., and D. Berghmans, A broad perspective on automated CME tracking: towards higher level space weather forecasting. Edited by N. Gopalswamy, R. Mewaldt, and J. Torsti, Solar Eruptions and Energetic Particles, 165, Washington D.C: AGU Press, Geophysical Monograph Series, 33-41, 2006.

Rodriguez, L., A.N. Zhukov, C. Cid, Y. Cerrato, E. Saiz, H. Cremades, S. Dasso, M. Menvielle, A. Aran, C. Mandrini, et al., Three frontside full halo coronal mass ejections with a nontypical geomagnetic response, Space Weather, 7, S06003, DOI: 10.1029/2008SW000453, 2009.

Rouillard, A.P., M. Lockwood, and I. Finch, Centennial changes in the solar wind speed and in the open solar flux, J. Geophys. Res., 112, A05103, DOI: 10.1029/2006JA012130, 2007.

Šafránková, J., Z. Němeček, L. Přech, J. Šimůnek, D. Sibeck, and J.-A. Sauvaud, Variations of the flank LLBL thickness as response to the solar wind dynamic pressure and IMF orientation, J. Geophys. Res., 112, A07201, DOI: 10.1029/2006JA011889, 2007.

Saiz, E., C. Cid, and Y. Cerrato, Forecasting intense geomagnetic activity using interplanetary magnetic field data, Ann. Geophys., 26, 3989-3998, 2008.

Schunk, R.W., and L. Zhu, Response of the ionosphere-thermosphere system to magnetospheric processes, J. Atmos. Solar Terr. Phys., 70 (18), 2358-2373, DOI: 10.1016/j.jastp.2008.07.003, 2008.

Schwenn, R., Space weather: the solar perspective, Living Rev. Sol., 3, 5-72, lrsp-2006-2, 2006.

Scopke, N., A General Relation between the Energy of Trapped Particles and the Disturbance Field near the Earth, J. Geophys. Res., 71, 3125-3130, 1966.

Søraas, F., K. Aarsnes, K. Oksavik, M.I. Sandanger, D.S. Evans, and M.S. Greer, Evidence for particle injection as the cause of Dst reduction during HILDCAA events, J. Atmos. Solar-Terr. Phys., 66 (2), 177-186, DOI: 10.1016/j.jastp.2003.05, 2004.
Stamper, R., M. Lockwood, M.N. Wild, and T.D.G. Clark, Solar causes of the long-term increase in geomagnetic activity, $J$. Geophys. Res., 104 (A12), 28325-28342,

DOI: $10.1029 / 1999 J A 900311,1999$.

Stauning, P., A new index for the interplanetary merging electric field and geomagnetic activity: application of the unified polar cap indices, Space Weather, 5, S09001, DOI: 10.1029/2007SW000311, 2007.

Stauning, P., The Polar Cap PC indices: relations to solar wind and global disturbances. Edited by M. Lazar, Exploring the Solar Wind, InTech, Chap. 16, 357-398, DOI: 10.5772/37359, 2012.

Stauning, P., O.A. Troshichev, and A. Janzhura, The Polar Cap (PC) index: relations to solar wind parameters and global activity level, J. Atmos. Solar-Terr. Phys.,

DOI: 10.1016/j.jastp.2008.09.028, 2008.

Steinhilber, F., J.A. Abreu, J. Beer, and K.G. McCracken, Interplanetary magnetic field during the past 9300 years inferred from cosmogenic radionuclides, J. Geophys. Res., 115, A01104, DOI: 10.1029/2009JA014193, 2010.

Svalgaard, L., Geomagnetic activity: dependence on solar wind parameters. Edited by A. Zirker, Coronal Holes and High Speed Wind Streams, Colorado Assoc. Univ. Press, 371-441, 1978.

Svalgaard, L., and E.W. Cliver, The $I D V$ index: its derivation and use in inferring long-term variations of the interplanetary magnetic field strength, J. Geophys. Res., 110, A12103, DOI: 10.1029/2005JA011203, 2005.

Svalgaard, L., and E.W. Cliver, Interhourly variability index of geomagnetic activity and its use in deriving the long-term variation of solar wind speed, J. Geophys. Res., 112, A10111, DOI: 10.1029/2007JA012437, 2007.

Svalgaard, L., E.W. Cliver, and P. Le Sager, Determination of interplanetary magnetic field strength, solar wind speed, and EUV irradiance. Edited by A. Wilson, International Solar Cycle Studies Symposium, Tatranska Lomnica, Slovak Republic, Proceedings (ESA SP-535), 15-24, 2003.

Svalgaard, L., E.W. Cliver, and P. Sager, IHV: a new long-term geomagnetic index, Adv. Space Res., 34, 1-2, DOI: 10.1016/j.asr.2003.01.029, 2004.

Takahashi, S., T. Iyemori, and M. Takeda, A simulation of the storm time ring current, Planet. Space Sci., 38 (9), 1133-1141, DOI: 10.1016/0032-0633(90)90021-H, 1990.

Teodosiev, D., E. Yordanova, P. Nenovski, T. Nikolova, D. Danov, G. Crowley, L. Baddeley, and S. Buchert, Ion Temperature Distribution in the High-Latitude Region, Comptes Rendus (EISCAT UHF Radar Observations). What is the Field-aligned Currents Influence?, Acad. Bul. Sci., 64 (5), 729-736, 2011.

Thomson, A.W.P., C.T. Gaunt, P. Cilliers, J.A. Wild, B. Opperman, L.A. McKinnell, P. Kotze, C.M. Ngwira, and S.I. Lotz, Present dat challenges in understanding the geomagnetic hazard to national power grids, Adv. Space Res., 45, 1182-1190, 2010.

Tóth, G., I.V. Sokolov, T.I. Gombosi, D.R. Chesney, C.R. Clauer, D.L. DeZeeuw, K.C. Hansen, K.J. Kane, W.B. Manchester, R.C. Oehmke, et al., Space Weather Modeling Framework: a new tool for the space community, J. Geophys. Res., 110, A12226, DOI: 10.1029/2005JA011126, 2005.

Troshichev, O.A., Ground-based Monitoring of the Solar Wind Geoefficiency. Edited by M. Lazar, Exploring the Solar Wind, InTech, Chap. 15, 337-356, DOI: 10.5772/38227, ISBN 978-95351-0339-4, http://www.intechopen.com/books/exploring-the-solarwind/ground-based-monitoring-of-the-solar-wind-geoefficiency, 2012.

Troshichev, O.A., V.G. Andrezen, S. Vennerstrøm, and E. FriisChristensen, Magnetic activity in the polar cap - a new index, Planet. Space Sci., 36, 1095-1102, 1988.

Troshichev, O.A., A. Janzhura, and P. Stauning, Unified PCN and PCS indices: method of calculation, physical sense and dependence on the IMF azimuthal and northward components, $J$. 
Geophys. Res., 111, A05208, DOI: 10.1029/2005JA011402, 2006.

Tsurutani, B.T., and W.D. Gonzalez, The cause of high-intensity long-duration continuous AE activity (HILDCAAs): interplanetary Alfvén wave trains, Planet. Space Sci., 35, 405-412, 1987.

Tsurutani, B., A. Mannucci, B. Iijima, M.A. Abdu, J.H.A. Sobral, W. Gonzalez, F. Guarnieri, T. Tsuda, A. Saito, and K. Yumoto, et al., Global dayside ionospheric uplift and enhancement associated with interplanetary electric fields, J. Geophys. Res., 109, A08302, DOI: 10.1029/2003JA010342, 2004.

Turner, N.E., W. Douglas Cramer, S.K. Earles, and B.A. Emery, Geoefficiency and energy partitioning in CIR-driven and CMEdriven storms, J. Atmos. Solar Terr. Phys., 71 (10-11), $1023-$ $1031,2009$.

Twitty, C., T.D. Phan, G. Paschmann, B. Lavraud, H. Rème, and M. Dunlop, Cluster survey of cusp reconnection and its IMF dependence, Geophys. Res. Lett., 31, L19808, DOI: 10.1029/2004GL020646, 2004.

Usoskin, I.G., K. Mursula, S.K. Solanki, M. Schüssler, and G. Kovaltsov, A physical reconstruction of cosmic ray intensity since 1610, J. Geophys. Res., 107 (11), 1374, DOI: 10.1029/2002JA009343, 2002.

Usoskin, I.G., S.K. Solanki, and G.A. Kovaltsov, Grand minima and maxima of solar activity: new observational constraints, $A \& A$, 471, 301-309, DOI: 10.1051/0004-6361:20077704, 2007.

Valach, F., P. Hejda, and J. Bochníček, Geoeffectiveness of XRA events associated with RSP II and/or RSP IV estimated using the artificial neural network, Stud. Geophys. Geod., 51 (4), 551-562, DOI: 10.1007/s11200-007-0032-5, 2007.

Valach, F., M. Revallo, J. Bochníček, and P. Hejda, Solar energetic particle flux enhancement as a predictor of geomagnetic activity in a neural network-based model, Space Weather, 7, S04004, DOI: 10.1029/2008SW000421, 2009.

Vasyliunas, V.M., The interrelationship of magnetospheric processes. Edited by B.M. McCormac, Earth's Magnetospheric Processes, Dordrecht, Holland: D. Reidel Pub, 29-38, 1972.
Vasyliunas, V.M., Reinterpreting the Burton-McPherron-Russell equation for predicting Dst, J. Geophys. Res., 111, A07S04, DOI: 10.1029/2005JA011440, 2006.

Wang, C.B., J.K. Chao, and C.H. Lin, Influence of the solar wind dynamic pressure on the decay and injection of the ring current, J. Geophys. Res., 108 (9), 1341, DOI: 10.1029/2003JA009851, 2003

Wang, R., and J. Wang, Investigation of the cosmic ray ground level enhancements during solar cycle 23, Adv. Space Res., 38, 489492, 2006.

Wanliss, J.A., and K.M. Showalter, High-resolution global storm index: Dst versus SYM-H, J. Geophys. Res., 111, A02202, DOI: 10.1029/2005JA011034, 2006.

Weigel, R.S., Solar wind density influence on geomagnetic storm intensity, J. Geophys. Res., 115, A09201, DOI: 10.1029/2009JA015062, 2010.

Weimer, D.R., Improved ionospheric electrodynamic models and application to calculating Joule heating rates, J. Geophys. Res., 110, A05306, DOI: 10.1029/2004JA010884, 2005.

Weygand, J.M., and R.L. McPherron, Dependence of ring current asymmetry on storm phase, J. Geophys. Res., 111, A11221, DOI: 10.1029/2006JA011808, 2006.

Wild, J.A., S.E. Milan, C.J. Owen, J.M. Bosqued, M. Lester, D.M. Wright, H. Frey, C.W. Carlson, A.N. Fazakerley, and H. Remè, The location of the open-closed magnetic field line boundary in the dawn sector auroral ionosphere, Ann. Geophys., 22, 36253639, SRef-ID: 1432-0576/ag/2004-22-3625, 2004.

Zhang, X.X., C. Wang, T. Chen, Y.L. Wang, A. Tan, T.S. Wu, G.A. Germany, and W. Wang, Global patterns of Joule heating in the high-latitude ionosphere, J. Geophys. Res., 110, A12208, DOI: 10.1029/2005JA011222, 2005.

Zurbuchen, T.H., and I.G. Richardson, In-Situ Solar Wind and Magnetic Field Signatures of Interplanetary Coronal Mass Ejections, Space Sci. Rev., 123, 31-43, DOI: $10.1007 / \mathrm{s} 11214-006-9010-4,2006$.

Cite this article as: Saiz E, Cerrato Y, Cid C, Dobrica V, Hejda P, et al.: Geomagnetic response to solar and interplanetary disturbances. J. Space Weather Space Clim., 2013, 3, A26. 